\title{
Polymer-bound haloate(I) anions by iodine(III)-mediated oxidation of polymer-bound iodide: synthetic utility in natural product transformations
}

\author{
Andreas Kirschning, ${ }^{\mathrm{a} *}$ Eike Kunst, ${ }^{\mathrm{a}}$ Monika Ries, ${ }^{\mathrm{c}}$ Lars Rose, ${ }^{\mathrm{c}}$ Andreas Schönberger, ${ }^{\mathrm{c}}$ and \\ Rudolf Wartchow \\ ${ }^{a}$ Institut für Organische Chemie, Universität Hannover, Schneiderberg 1B, D-30167 Hannover, \\ Germany \\ ${ }^{b}$ Institut für Anorganische Chemie, Universität Hannover, Callinstraße 9, D-30167 Hannover, \\ Germany \\ ${ }^{c}$ Institut für Organische Chemie, Technische Universität Clausthal, Leibnizstraße 6, 38678 \\ Clausthal-Zellerfeld, Germany \\ E-mail: andreas.kirschning@oci.uni-hannover.de
}

\section{Dedicated to Professor Anastassios Varvoglis on the occasion of his $65^{\text {th }}$ birthday}

(received 24 Jan 03; accepted 07 Apr 03; published on the web 08 May 03)

\begin{abstract}
A set of polymer-attached hypervalent iodate(I) complexes were prepared from polymer-bound iodide anion by ligand transfer of acetate and trifluoro acetate present in the corresponding iodine(III) reagents onto the iodide anion. The synthetic versatility of these polymer-bound reagents in terms of efficacy and ease of workup is demonstrated for selected examples in natural product synthesis and natural product derivatization. Thus, iodoacetoxylation of glycals is the initial step for the preparation of two deoxygenated disaccharides which are part of the carbohydrate units of the landomycins. In a second example, a one-pot multistep rearrangement of the decanolide decarestrictine $\mathrm{D}$ backbone is shown which is initiated by iodotrifluoroacylation of the olefinic double bond.
\end{abstract}

Keywords: Natural products, decanolide, glycosides, polymer-bound reagents, hypervalent iodine reagents, rearrangement

\section{Introduction}

Cohalogenation of alkenes constitutes one of the most important classes of reaction used to form a carbon heteroatom bond in a regio-, chemo- and stereoselective manner. ${ }^{1}$ Among this class of reactions, haloacylations, haloazidations and the 1,2-addition of iodine isocyanate are of 
particular relevance. Recently, we described a new class of anionic reagents, namely haloate(I) complexes, which synthetically behave like acylated hypohalite or iodoazide. The development of this new group of reagents was based on the observation that hypervalent iodine reagents ${ }^{2}$ in the oxidation state III are able to oxidize iodide and bromide anions by ligand transfer from the hypervalent reagent onto the halide anion (Scheme 1). ${ }^{3,4}$ The preparation of the acylated hypoiodite by this route proved to be superior to the thermal decomposition of aryl iodine diacetate which proceeds via three mechanisms. One of these routes was the nucleophilic displacement within an ion pair to give aryl acetate and acylated hypoiodite. ${ }^{5}$ In another study, Varvoglis and coworkers showed that 4-nitrophenyl hypoiodite can be prepared by treatment of iodine with phenyliodine(III) diphenolate. ${ }^{6}$

The potential of these reagents was extended by immobilizing them onto polystyrene support via ion exchange. Thus, conventional anion exchange resins loaded with bromide $\mathbf{1}$ or iodide $\mathbf{2}$, respectively, were oxidized by various hypervalent iodine reagents to yield resins $\mathbf{3}-\mathbf{6}$ which were purified by filtration and washing in order to remove traces of iodobenzene and excess of the iodoso reagent. Alternatively, bisazidoiodate(I) anion 6 can be prepared by ligand exchange of bisacetoxy anion 4 using trimethylsilyl azide. The polymer-bound ate(I) complexes can be applied for various synthetic purposes. E. g., reagent $\mathbf{3}$ was employed for the bromoacetoxylation of alkenes and the oxidation of alcohols. ${ }^{7}$ In addition, reagents $\mathbf{3}-\mathbf{6}$ were successfully employed in iodoacylation and iodoazidation of alkenes. ${ }^{8,9}$ Finally, electrophilic activation of phenylthio groups can be achieved with polymer-bound reagent $5^{10}$

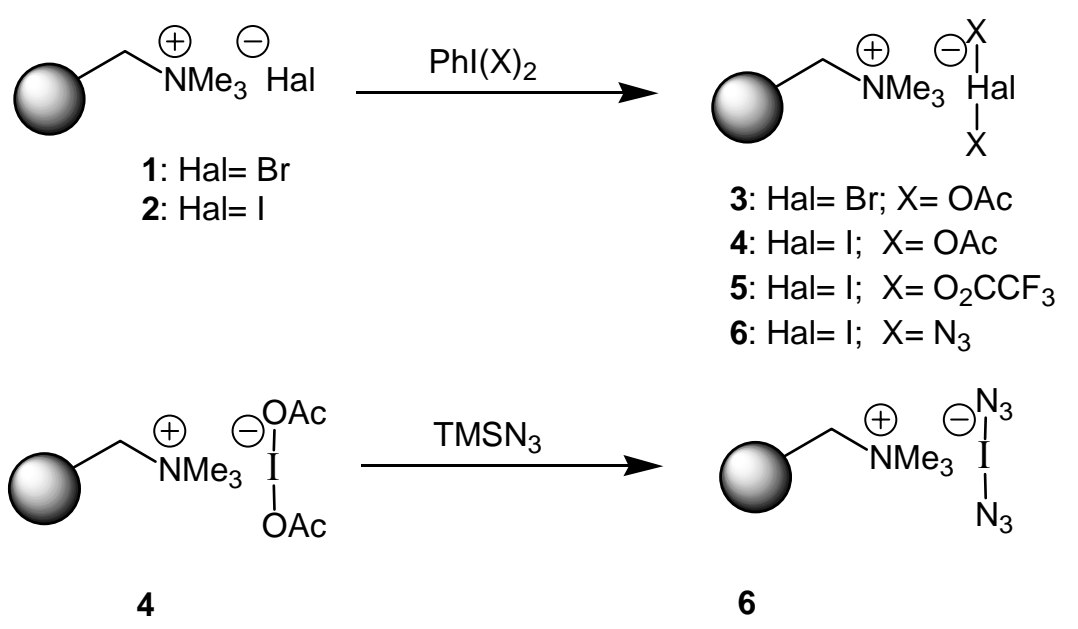

\section{Scheme 1}

It is generally accepted that the synthetic power of new reagents becomes particularly evident when applications for the synthesis of complex molecules and natural products can be developed. In this article the utility of these reagents is extended to applications of natural product transformations. 


\section{Results and Discussion}

\section{Transformations in oligosaccharide synthesis}

Glycoconjugates that are composed of an (oligo)deoxysugar portion and an aglycon are widely distributed in nature and are of wide clinical importance. ${ }^{11}$ Importantly, alterations of the saccharide structures can result in improved biological activity, in particular against drugresistant microorganisms. However, preparation of these glycoconjugates is still a challenging topic. In view of the importance and success of solid-phase chemistry various polymer-supported syntheses of oligosaccharides including deoxysugar analogues ${ }^{12}$ have been developed. ${ }^{13}$

In conjunction with our research activities in this field, ${ }^{14}$ polymer-bound haloate(I) complexes 3 - 6 were used for the polymer-assisted solution phase synthesis of deoxysugarbased glycoconjugates. In the context of deoxyglycoside synthesis, 2-iodo-glycosyl acetates are excellent glycosyl donors which can be activated for glycosidation with Lewis acids such as TMSOTf. Earlier studies had proven, that iodate(I)-complexes may promote 1,2functionalization of glycals under very mild conditions and with excellent yields. ${ }^{15}$
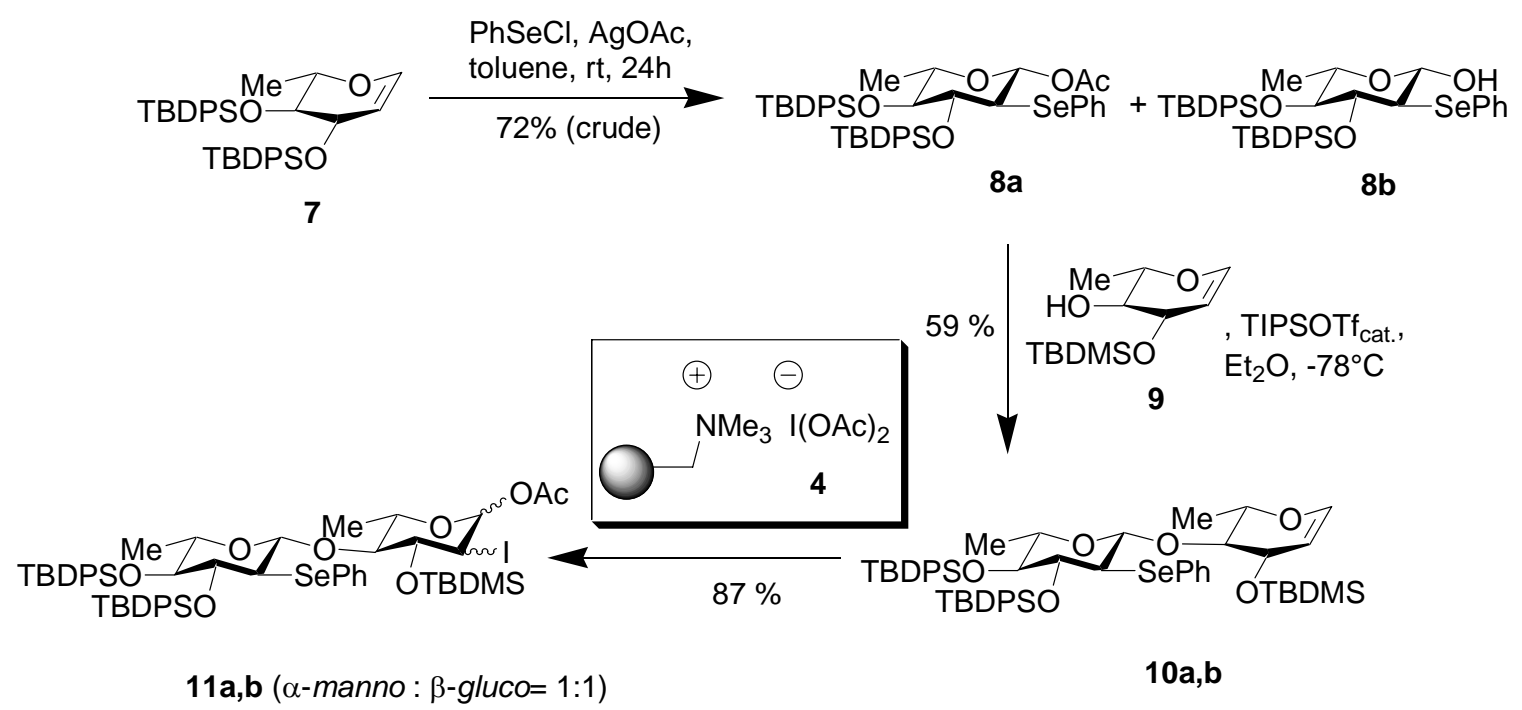

\section{Scheme 2}

As part of a synthesis directed towards the carbohydrate portion of the angucycline antibiotic landomycin, ${ }^{16}$ it became necessary to prepare 2-seleno glycosyl acetate $8 \mathbf{a}$ by following the method first described by Beau and coworkers. ${ }^{17}$ Thus, glycal 7 was treated with phenylselenyl chloride in the presence of silver acetate to yield the 1,2-addition product 8a as a single isomer. However at this stage it was difficult to prove the $\beta$-gluco-configuration of product 8a because the coupling constants $J$ for the ring protons in the ${ }^{1} \mathrm{H}$ NMR-spectrum are not diagnostic $\left(J_{1,2}=\right.$ $4.0 \mathrm{~Hz}, J_{2,3}=3.6 \mathrm{~Hz}, J_{3,4}=3.6 \mathrm{~Hz}, J_{4,5}$ not determined). Furthermore, the acetyl group in pyranosyl acetate 8a turned out to be very sensitive and was hydrolyzed upon attempted purification on silica gel which yielded pyranose 8b. In accordance with the observations by 
Beau and Perez, the $\beta$-gluco configured isomer was formed predominantly. Thus, the crude mixture was directly transferred into disaccharides by activation of the glycosyl acetate moiety with TIPS-triflate ${ }^{18}$ in the presence of glycal 9. As a result disaccharides 10a,b were formed which contains a glycal moiety (Scheme 2). At this point, we tested the utility of the polymerbound hypervalent iodate(I) reagent 4 as the resulting 2-iodo-glycosyl acetate is ideally suited for further glycosidations (vide supra). The functionalization of the enol ether bond in disaccharide of $\beta$-gluco-10a is a particular challenge, because the phenylseleno group is present in the molecule which is prone to oxidation. Iodonium cations are soft electrophiles which can coordinate to the seleno group inducing undesired side reactions. Polymer-bound iodate(I) complex 4 turned out to be a very mild reagent for the 1,2-iodoacetoxylation of the glycal double bond yielding glycosyl acetates 11a,b as a diastereomeric mixture $(\alpha-$ manno : $\beta$-gluco=1:1).

In the following this concept was further extended to disaccharide 19, again using polymerbound iodate(I) reagent 4 as the key reagent for the 1,2-functionalization of a glycal olefinic double bond (Scheme 3). In addition, polymer-bound silyl triflate $\mathbf{1 4}{ }^{19}$, used as an activator of the anomeric acyl group was used in this example.

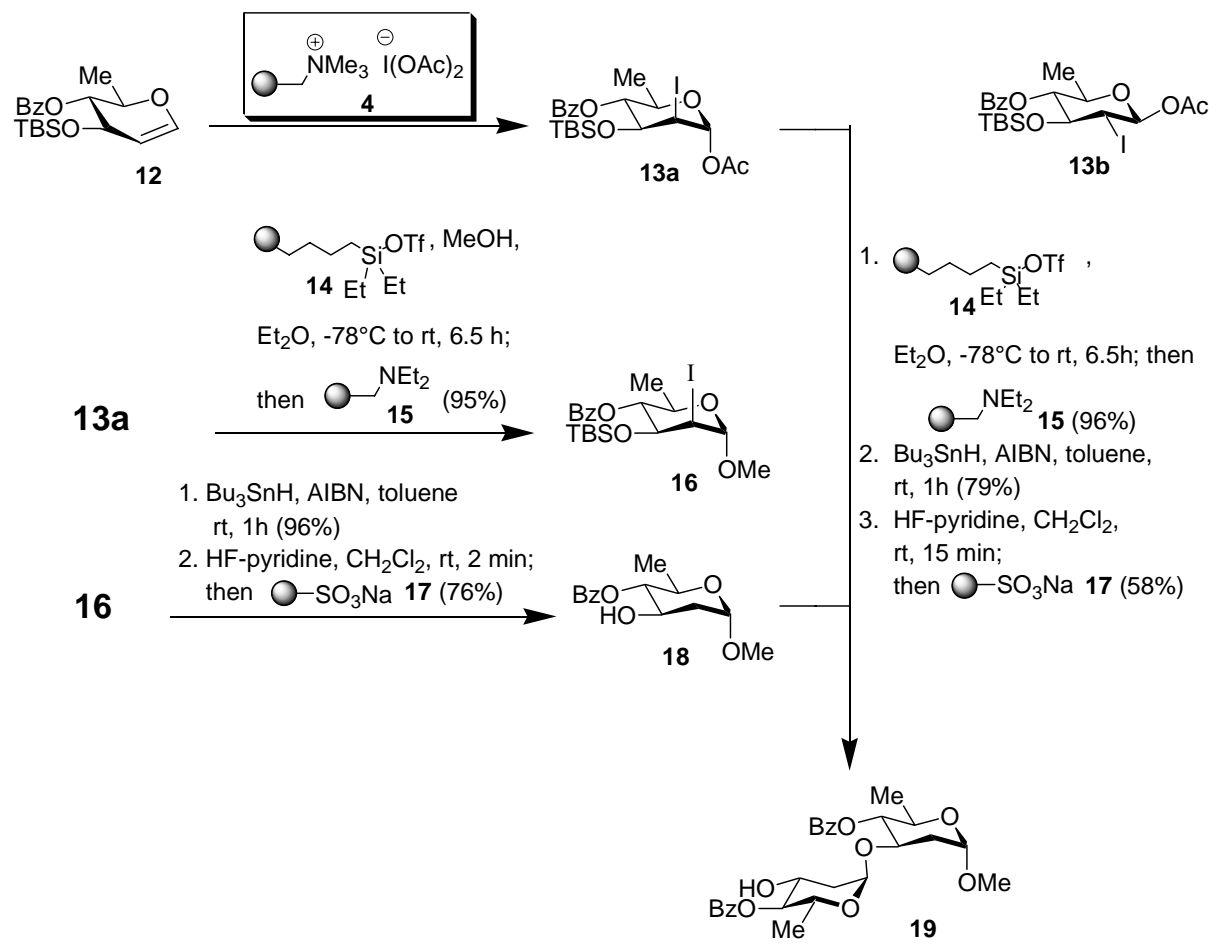

\section{Scheme 3}

Thus, activation of the D-glucal derivative 12 with reagent 4 yielded the separable diastereomeric mixture of 2-iodo-glycosyl acetates 13a,b. Synthesis was continued with isomer 13 a by treatment with silyltriflate 14 and methanol as acceptor which led to methyl glycoside 16 . Polymer-bound amine (Amberlyst A-21) 15 was added at the end of the synthesis in order to 
remove traces of $\mathrm{CF}_{3} \mathrm{SO}_{2} \mathrm{H}$. Further modification included deiodination and $O$-desilylation. The former step was conducted under conventional deiodination conditions $\left(\mathrm{Bu}_{3} \mathrm{SnH}\right.$, AIBN). Subsequently, desilylation was achieved using the HF-pyridine complex. ${ }^{20}$ Excess desilylating reagent was removed with Amberlite A-200 $\left(\mathrm{Na}^{+}\right.$-form) 17. This scavenging reagent turned out to be very efficient for trapping protonated pyridine as well as the fluoride anion as its sodium salt. Both solids were simply filtered off and methyl glycoside 18 was isolated in pure form. Now, the stage was set for a second glycosidation step using olivosyl acetate 13a and polymerbound silyl triflate 14. After neutralization, filtration and removal of the solvent in the usual manner, disaccharide was deiodinated and desilylated as described before to yield the target disaccharide 19. These sequences are a clear proof for the highly versatile synthetic properties of iodate(I) complex 4.

\section{Polymer-assisted one-pot multistep rearrangement of decarestrictine D}

In a second set of experiments, we envisaged synthetic multistep one-pot derivatizations of pharmaceutically important natural products using polymer-bound haloate(I) reagents. Recently, it became clear that combinatorial derived compound libraries are often large in size but lack quality because of defficiencies in structural uniqueness and diversity. This also includes multifunctionality and chirality. Due to the complexity of natural products only a few efforts have been made to synthesize libraries of natural products and analogues. ${ }^{21}$ One of the best examples in this context are the sarcodyctynes for which the group of Nicolaou developed a solid-phase approach. ${ }^{22}$ Alternatively, the inherent biological potency, the structural complexity and the threedimensional character of most natural products make them ideal templates for the creation of compound libraries which fulfill the requirements for quality. One approach is the stepwise "decoration" of individual functional groups on the chiral natural product-derived template employing combinatorial chemistry techniques. In this respect, carbohydrates served as templates in various applications, lately. ${ }^{23}$ Alternatively but rarely tested, natural products can be the starting point of cascade-type transformations, which may include rearrangments of the carbon backbone. ${ }^{24}$ Consequently, new natural product like systems with some of the chiral centers kept intact are generated for which structurally no reminiscence is desrcibed from natural sources.

Following this latter approach it was believed that as natural products are precious and are often available in only minute quantities, polymer-bound reagents like the electrophilic haloate(I) complexes $\mathbf{4}$ and $\mathbf{5}$ are ideally suited to carry out multistep transformations on a small scale. This technique allows simplification of work-up procedures and loss of material is reduced to a minimum.

As a multifunctional natural product the ten-membered lacton decarestrictine D $2 \mathbf{2 0}^{25}$ was chosen which among other decanolides was isolated from the fermentation broth of Penicillium corylophilum, simplicissimum, and independently from the fungus polyphorus tuberaster ${ }^{26}$ it is

an important new member of the growing class of ten-membered lactones of natural origin. ${ }^{27}$ It was demonstrated that decanolide $\mathbf{2 0}$ is a potent in vivo inhibitor of de novo cholesterol 
biosynthesis. Analyzing the donor and acceptor properties of all functional groups of decarestrictine, it is obvious that nucleophilic groups like the hydroxy group and the ester oxygen atoms and a masked carboxylate functionality prevail. The olefinic double bond, however can easily act as a counterpart if approached by an electrophilic species. Here, either the intermediate cation or the 1,2-addition products could initiate a cascade reaction.

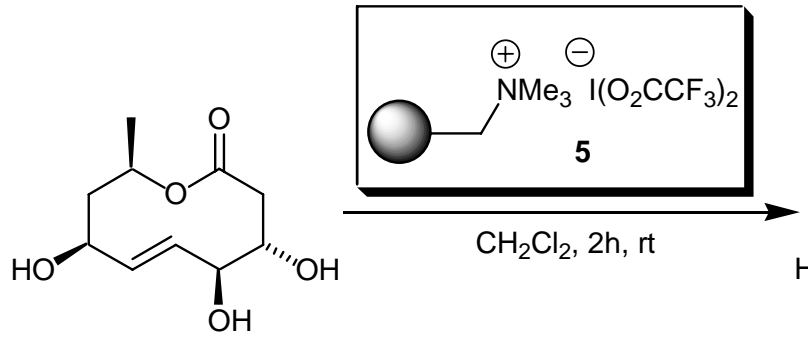

decarestrictine D 20

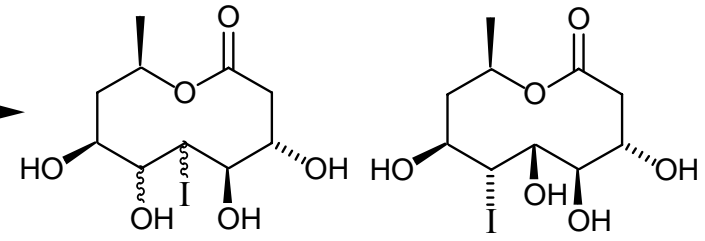

21a,b
22

\section{Scheme 4}

In an initial phase of the project, it was first tried to evaluate the reactivity of the olefinic double bond in decarestrictine D $\mathbf{2 0}$ by treatment of the unprotected natural product with polymer-bound bis-trifluoacetoxy iodate(I) 5 (Scheme 4). Purification on silica-gel afforded one fraction which contained a mixture of 1,2-functionalized products of which diastereomers 21a and $21 \mathbf{b}$ could be analytically deduced. Regio- and stereoisomer 22 was isolated as a second fraction. The formation of iodo hydrines can be rationalized by assuming that the primary 1iodo-2-trifluoroacylation products hydrolyze upon work-up.

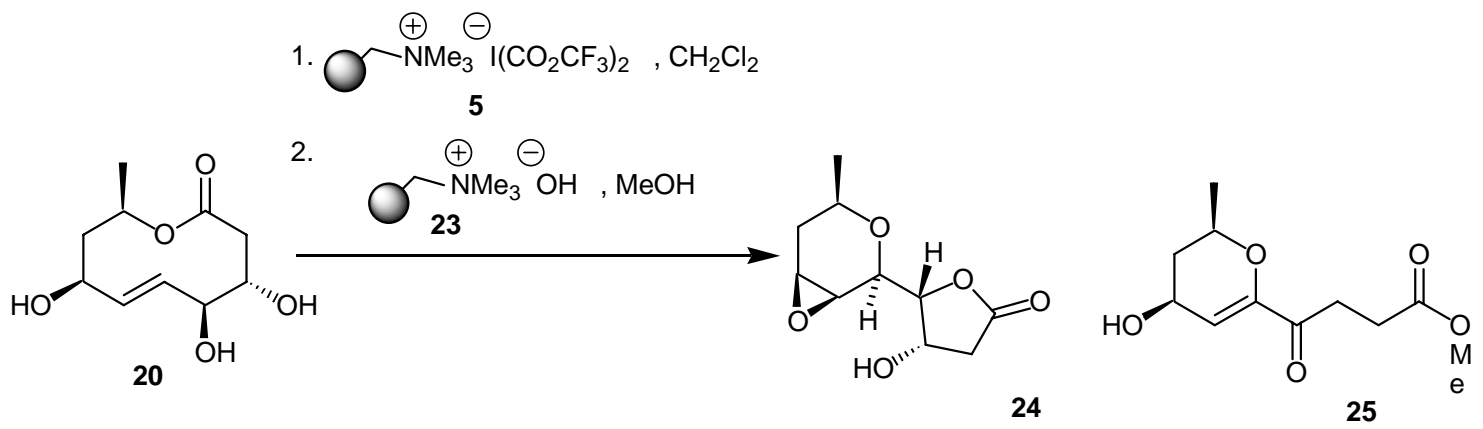

\section{Scheme 5}

Based on these preliminary results we reacted unprotected decarestrictine D with polymerbound bis(trifluoroacetoxy)iodate(I) 5 (Scheme 5). As expected the intermediate addition products turned out to be rather labile (refer also to the results summarized in Scheme 4) so that the crude material was used for the next step. Indeed, when it was treated with polymer-bound hydroxide 23 in methanol two major products were identified by t.l.c. (about $50 \%$ crude). After 
filtration and careful chromatographic purification, the natural product like compounds 24 and 25 were isolated. Obviously, the second reaction was accompanied with substantial rearrangement of the decanolide backbone. Structural evidence for the two products was gained from detailed NMR-spectroscopic analysis. Thus, a series of COSY, selective NOESY (Table 1), HMBC and selective TOCSY-experiments were carried out to assign the ${ }^{1} \mathrm{H}$ and ${ }^{13} \mathrm{C}$ signals as well as the relative stereochemistry between $5-\mathrm{H}$ and $6-\mathrm{H}$ in epoxylactone 24 . Final structural proof was obtained from an X-ray analysis (Figure 1, Table 2).

Table 1. Observed NOEs in compound 24

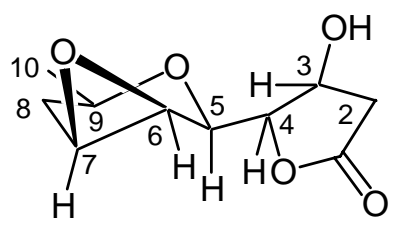

24

\begin{tabular}{cc}
\hline & Nuclear Overhauser Effects \\
\hline $10-\mathrm{H}$ & $8-\mathrm{H}_{\mathrm{eq}}, 8-\mathrm{H}_{\mathrm{ax}}, \mathrm{H}-9$ \\
$9-\mathrm{H}$ & $5-\mathrm{H}, 8-\mathrm{H}_{\mathrm{eq}}(+), 8-\mathrm{H}_{\mathrm{ax}}(-), 10-\mathrm{H}$ \\
$7-\mathrm{H}$ & $6-\mathrm{H}, 8-\mathrm{H}_{\mathrm{eq}}$ \\
$6-\mathrm{H}$ & $5-\mathrm{H}, 9-\mathrm{H}$ \\
$5-\mathrm{H}$ & $4-\mathrm{H}, 9-\mathrm{H}, 6-\mathrm{H}, 3-\mathrm{OH}$ \\
$3-\mathrm{H}$ & $4-\mathrm{H}, 6-\mathrm{H}, 3-\mathrm{OH}, 2-\mathrm{H}_{\mathrm{eq}}, 2-\mathrm{H}_{\mathrm{ax}}$ \\
\hline
\end{tabular}

It is worth to note that the relative orientation of the pyran ring and the lactone are opposite to each other in solution and in the crystal.

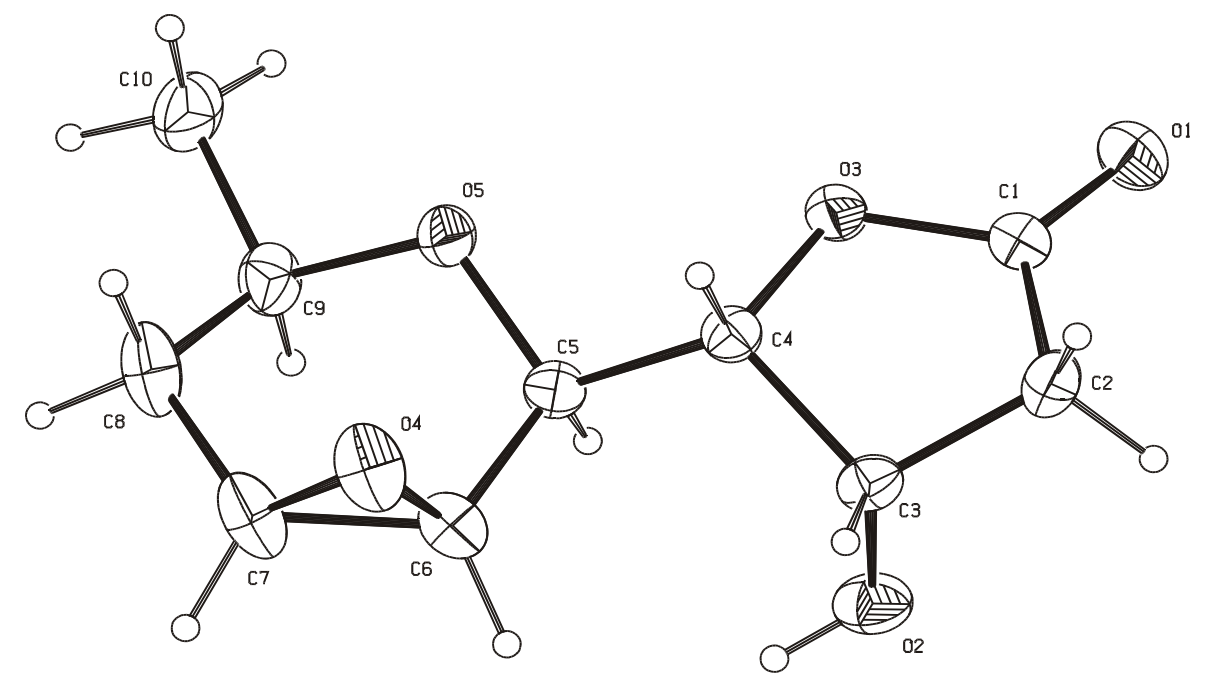

Figure 1. ORTEP representation of $\mathbf{2 4}$. 
Table 2. Significant interatomic bond distances and angles in 24

\begin{tabular}{|c|c|c|c|}
\hline \multicolumn{4}{|c|}{ Bond lengths [A] } \\
\hline $\mathrm{O}(1)-\mathrm{C}(1)$ & $1.204(2)$ & $C(2)-C(3)$ & $1.501(2)$ \\
\hline $\mathrm{O}(2)-\mathrm{C}(3)$ & $1.413(2)$ & $C(3)-C(4)$ & $1.529(2)$ \\
\hline $\mathrm{O}(3)-\mathrm{C}(1)$ & $1.344(2)$ & $C(4)-C(5)$ & $1.506(2)$ \\
\hline $\mathrm{O}(3)-\mathrm{C}(4)$ & $1.456(2)$ & $C(5)-C(6)$ & $1.491(2)$ \\
\hline $\mathrm{O}(4)-\mathrm{C}(6)$ & $1.426(2)$ & $C(6)-C(7)$ & $1.446(2)$ \\
\hline $\mathrm{O}(4)-\mathrm{C}(7)$ & $1.439(2)$ & $C(7)-C(8)$ & $1.485(3)$ \\
\hline $\mathrm{O}(5)-\mathrm{C}(5)$ & $1.419(2)$ & $C(8)-C(9)$ & $1.510(3)$ \\
\hline $\mathrm{O}(5)-\mathrm{C}(9)$ & $1.437(2)$ & $\mathrm{C}(9)-\mathrm{C}(10)$ & $1.509(3)$ \\
\hline$C(1)-C(2)$ & $1.471(3)$ & & \\
\hline \multicolumn{4}{|c|}{ Bonding angles $\left[{ }^{\circ}\right]$} \\
\hline $\mathrm{C}(1)-\mathrm{O}(3)-\mathrm{C}(4)$ & $109.79(12)$ & $\mathrm{C}(5)-\mathrm{C}(4)-\mathrm{C}(3)$ & $115.14(13)$ \\
\hline $\mathrm{C}(6)-\mathrm{O}(4)-\mathrm{C}(7)$ & $60.65(12)$ & $\mathrm{O}(5)-\mathrm{C}(5)-\mathrm{C}(6)$ & $112.41(13)$ \\
\hline $\mathrm{C}(5)-\mathrm{O}(5)-\mathrm{C}(9)$ & $111.10(13)$ & $\mathrm{O}(5)-\mathrm{C}(5)-\mathrm{C}(4)$ & $107.75(12)$ \\
\hline $\mathrm{O}(1)-\mathrm{C}(1)-\mathrm{O}(3)$ & $121.0(2)$ & $C(6)-C(5)-C(4)$ & $111.14(13)$ \\
\hline $\mathrm{O}(1)-\mathrm{C}(1)-\mathrm{C}(2)$ & $128.9(2)$ & $\mathrm{O}(4)-\mathrm{C}(6)-\mathrm{C}(7)$ & $60.11(12)$ \\
\hline $\mathrm{O}(3)-\mathrm{C}(1)-\mathrm{C}(2)$ & $110.10(14)$ & $\mathrm{O}(4)-\mathrm{C}(6)-\mathrm{C}(5)$ & $116.6(2)$ \\
\hline $\mathrm{C}(1)-\mathrm{C}(2)-\mathrm{C}(3)$ & $103.33(12)$ & $C(7)-C(6)-C(5)$ & $119.7(2)$ \\
\hline $\mathrm{O}(2)-\mathrm{C}(3)-\mathrm{C}(2)$ & $108.54(13)$ & $\mathrm{O}(4)-\mathrm{C}(7)-\mathrm{C}(6)$ & $59.25(10)$ \\
\hline $\mathrm{O}(2)-\mathrm{C}(3)-\mathrm{C}(4)$ & $110.34(12)$ & $\mathrm{O}(4)-\mathrm{C}(7)-\mathrm{C}(8)$ & $116.6(2)$ \\
\hline $\mathrm{C}(2)-\mathrm{C}(3)-\mathrm{C}(4)$ & $101.23(12)$ & $C(6)-C(7)-C(8)$ & 119.2(2) \\
\hline $\mathrm{O}(3)-\mathrm{C}(4)-\mathrm{C}(5)$ & $110.14(11)$ & $C(7)-C(8)-C(9)$ & 112.1(2) \\
\hline $\mathrm{C}(10)-\mathrm{C}(9)-\mathrm{C}(8)$ & $114.8(2)$ & $\mathrm{O}(5)-\mathrm{C}(9)-\mathrm{C}(10)$ & 107.7(2) \\
\hline $\mathrm{O}(3)-\mathrm{C}(4)-\mathrm{C}(3)$ & $103.52(11)$ & $\mathrm{O}(5)-\mathrm{C}(9)-\mathrm{C}(8)$ & $108.39(14)$ \\
\hline
\end{tabular}

Based on these data, it was possible to propose hypothetical mechanisms for the formation of rearranged products $\mathbf{2 4}$ and 25. After $\alpha$-attack of the iodonium cation to the olefinic double bond, intermediate 26 was either trapped by the hydroxy group at C-7 to yield oxirane 27 or alternatively by the 4-OH to furnish oxirane 28. Deprotonation of the 4-hydroxy group by the polymer-bound hydroxide anion initiated lactonization followed by ring closure of the intermediate alkoxy anion at C-9. The second route begins with methanolysis of 28 which creates the methyl ester. Hence, the alkoxy anion at C-9 cyclized to yield tetrahyropyran 29. In order to end up with dihydropyran 26 two elimination steps and a final tautomerization are required although the driving force of the process remains obscure. In order to get a more precise insight into the latter mechanism it will become necessary to search for additional byproducts. 


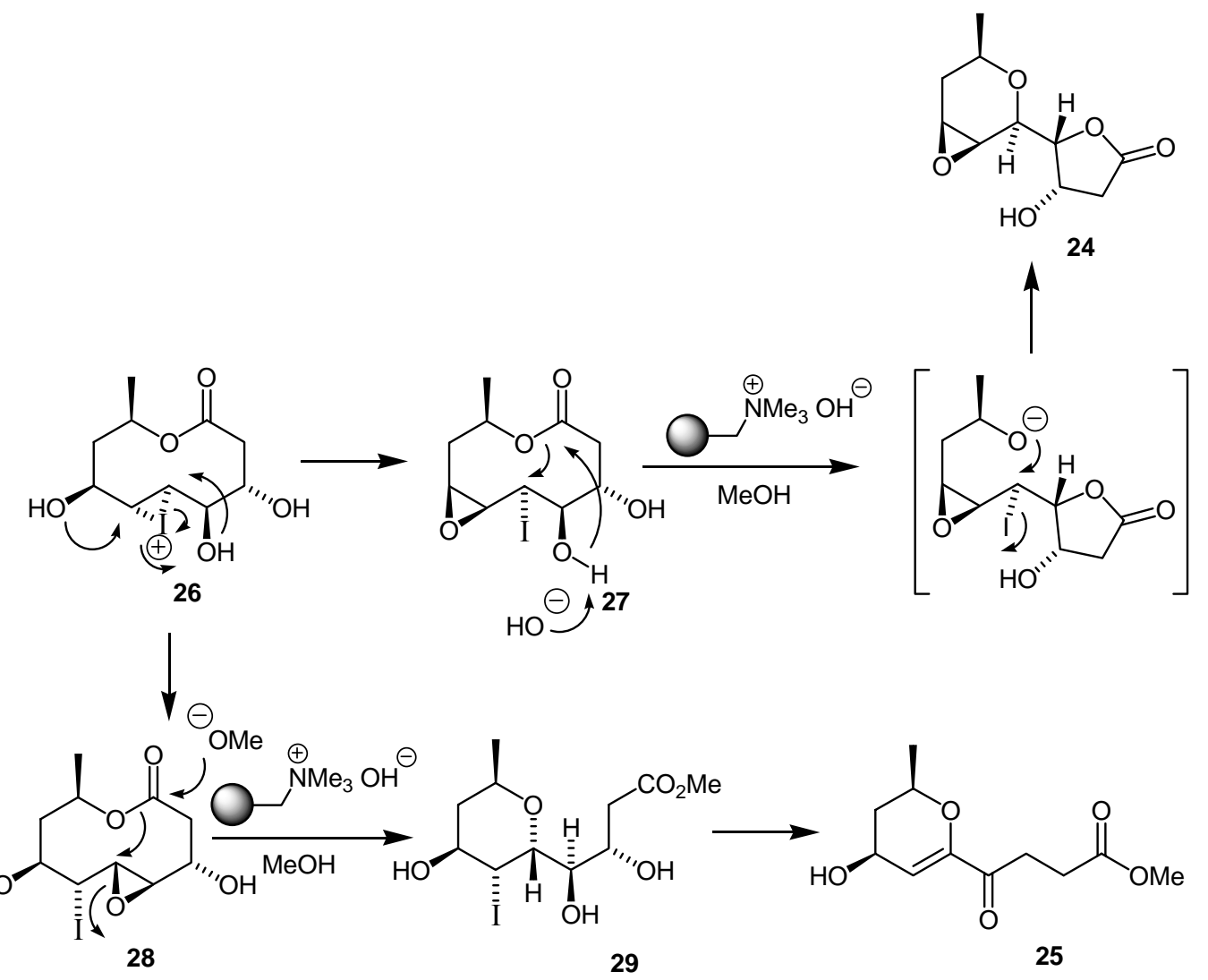

\section{Scheme 6}

\section{Conclusions}

In summary, it was demonstrated that the oxidation of polymer-bound iodide by hypervalent iodine reagents in the oxidation state III results in the formation of polymer-bound iodate(I) complexes. These are versatile synthetic tools for transformations in natural product chemistry.

\section{Experimental Section}

General Procedures. Melting Points were determined on a melting point apparatus from Büchi. Flash chromatography was performed on silica gel (Merck 60, 70-230 mesh). Thin layer chromatography was performed on aluminium backd plates of silica gel 60 F254 (Merck, 0.2 $\mathrm{mm}$ ) with the indicated eluent. NMR spectra were recorded on a Bruker ARX-400 (9.4 Tesla, 400.13 MHz for ${ }^{1} \mathrm{H}, 100.62 \mathrm{MHz}$ for ${ }^{13} \mathrm{C}$ and on a Bruker ARX-500 (500.13 MHz for ${ }^{1} \mathrm{H}, 125.03$ for ${ }^{13} \mathrm{C}$ ). If not otherwise noted, chemical shifts are reported relative to the internal standard (TMS: $\delta=0.0 \mathrm{ppm}$ ) for ${ }^{1} \mathrm{H}$ NMR and ${ }^{13} \mathrm{C}$ NMR. Coupling constants $(J$ in $\mathrm{Hz}$ ) are accurate to $+/-$ 
0.2 Hz. Mass spectra were recorded on a LC/MSD Series 1100, Hewlett Packard with the Kayak XA processing unit. Optical rotations $[\alpha]_{D}$ were measured with a Perkin Elmer 243B polarimeter (sodium line). Combustion analysis were carried out at the institute of pharmaceutical chemistry of the Technical University of Braunschweig.

Detailed descriptions for obtaining polymer-bound reagents 4 and 5 can be found in references $^{7,9,12}$. The preparation of glycals 7, 9 and $\mathbf{1 2}$ are reported in reference ${ }^{28}$ and in the literature cited therein. Their preparation is based on a procedure disclosed by Beau and coworkers. $^{17}$

Acetyl 3,4-bis-O-(tert-butyldiphenylmethylsilyl)-2,6-dideoxy-2-phenylseleno- $\beta$-L-glucohexopyranose 8a and 3,4-bis-O-(tert-butyldiphenylmethylsilyl)-2,6-dideoxy-2-phenylseleno$\beta$-L-gluco-hexopyranose (8b). A solution of glycal 7 (1.05 g, $1.7 \mathrm{mmol})$ in abs. toluene (50 $\mathrm{ml}$ ) was treated with $\mathrm{PheSeCl}$ (422 mg, $2.2 \mathrm{mmol}$ ) and AgOAc (435 mg, $2.6 \mathrm{mmol}$ ) at rt under nitrogen atmosphere. Stirring was continued for $3 \mathrm{~h}$ after which time the silver salts were filtered off. The solution was concentrated in vacuo. The crude material $(1.01 \mathrm{~g}, 1.23 \mathrm{mmol} ; 72 \%)$ obtained after gel filtration (silica gel; petroleum ether / ethyl acetate 12:1) can be used for the next step. For analytical purposes a small amount was purified by column chromatography (silica gel; petroleum ether / ethyl acetate 20:1).

1st fraction 8a. colorless oil; ${ }^{1} \mathrm{H}$ NMR $\left(400 \mathrm{MHz}, \mathrm{C}_{6} \mathrm{D}_{6}\right): \delta=7.70-7.10(\mathrm{~m}, 25 \mathrm{H}, \mathrm{Ar}), 6.32(\mathrm{~d}, J=$ $4.0 \mathrm{~Hz}, 1 \mathrm{H} \mathrm{1-H}), 4.39$ (dd, $J=3.6,3.6 \mathrm{~Hz}, 1 \mathrm{H}, 3-\mathrm{H}), 3.81$ (dd, $J=3.6,4.0 \mathrm{~Hz}, 1 \mathrm{H}, 2-\mathrm{H}), 3.64$ (m, 2H, 4-H, 5-H), 2.12 (s, 3H, OAc), 1.12 (d, J=7.2 Hz, 3H, 6-H), 0.97, 0.79 (2s, 18H, 2x tBu); ${ }^{13} \mathrm{C}-\mathrm{NMR}\left(100 \mathrm{MHz}, \mathrm{C}_{6} \mathrm{D}_{6}\right.$ ) $\delta: 169.5$ (s, OAc), 132.4, 132.3, 131.9, 131.8, 129.0 (s, Ar), 135.6, 135.4, 134.6, 134.5, 132.0, 128.8, 128.7, 128.6, 128.5, 126.6, 126.5, 126.4, 126.3, 126.2 (d, Ar), 91.0 (d, C-1), 72.4, 71.5, 70.8 (d, C-3, C-4, C-5), 44.6 (d, C-2), 26.2, 25.6 (2q, $\mathrm{CH}_{3}$ of $\left.t \mathrm{Bu}\right), 20.5$ (q, OAc), 19.0 (q, C-6), 18.5, $17.9(2 \mathrm{~s}, t \mathrm{Bu})$;

2nd fraction 8b. colorless solid; m.p.: $58^{\circ} \mathrm{C}$; $[\alpha]_{\mathrm{D}}{ }^{23}=+32.5^{\circ}\left(c=0.93, \mathrm{CHCl}_{3}\right) ;{ }^{1} \mathrm{H}$ NMR (400 $\left.\mathrm{MHz}, \mathrm{CDCl}_{3}\right): \delta=7.7-7.0(\mathrm{~m}, 25 \mathrm{H}, \mathrm{Ar}), 5.30(\mathrm{~d}, J=2.0,13.2 \mathrm{~Hz}, 1 \mathrm{H}, 1-\mathrm{H}), 4.78(\mathrm{dd}, J=2.0,2.0$ $\mathrm{Hz}, 1 \mathrm{H}, 3-\mathrm{H}), 3.96$ (q, J= 7.2 Hz, 1H, 5-H), 3.78 (d, J=13.2 Hz, 1H, OH), 3.54 (d, J=2.0 Hz, $1 \mathrm{H}, 4-\mathrm{H}), 3.14(\mathrm{dd}, J=2.0,2.0 \mathrm{~Hz}, 1 \mathrm{H}, 2-\mathrm{H}), 1.22$ (d, $J=7.2 \mathrm{~Hz}, 3 \mathrm{H}, 6-\mathrm{H}), 1.06,0.95(2 \mathrm{~s}, 18 \mathrm{H}$, 2x $t \mathrm{Bu}) ;{ }^{13} \mathrm{C}-\mathrm{NMR}\left(50 \mathrm{MHz}, \mathrm{CDCl}_{3}\right.$ ) $\delta: 133.4,132.9,132.3$ (s, Ar), 135.9, 135.8, 135.6, 132.6, 130.0, 129.9, 129.7, 129.0, 128.2, 127.8, 127.7, 127.6, 127.5, 126.8 (d, Ar),84.9 (d, C-1), 76.9, 75.9, 71.2 (d, C-3, C-4, C-5), 54.3 (d, C-2), 27.0, 26.9 (2q, $\mathrm{CH}_{3}$ of $\left.t \mathrm{Bu}\right), 19.1,18.9$ (2s, $\left.t \mathrm{Bu}\right), 16.6$ (q, C-6); combustion analysis calcd (\%) for $\mathrm{C}_{44} \mathrm{H}_{52} \mathrm{IO}_{4} \mathrm{SeSi}_{2}$ : C 67.73, H 6.72; found C 67.38, H 7.06 .

[3',4'-bis-O-(tert-butyldiphenylmethylsilyl)-2',6'-dideoxy-2'-phenylseleno- $\beta$-L-glucohexopyranosyl] (1-4)-1,5-anhydro-3-O-tert-butyldimethylsilyl-2,6-dideoxy-L-arabino-hex-1enitol (10a). A solution of glycosyl acetate 8a (1.45 g, $1.76 \mathrm{mmol})$ in abs. diethyl ether (50 ml) was cooled to $-78^{\circ} \mathrm{C}$ and TIPSOTf $(0.048 \mathrm{ml}, 55 \mathrm{mg}, 0.18 \mathrm{mmol})$ was added. After 10 minutes, glycal 9 (0.52 g, $2.1 \mathrm{mmol})$ was added. After $2 \mathrm{~h}$ the reaction mixture was hydrolyzed with aqueous bicarbonate and the aqueous phase was extracted with dichloromethane. The combined 
organic extracts were dried $\left(\mathrm{MgSO}_{4}\right)$, filtered and concentrated under reduced pressure. The crude material obtained was purified by column chromatography (silica gel; petroleum ether / ethyl acetate $30: 1)$.

${ }^{1 s t}$ fraction 10a. $(1.06 \mathrm{~g}, 1.05 \mathrm{mmol} ; 59 \%)$ : colorless solid; m.p.: $95^{\circ} \mathrm{C} ;[\alpha]_{\mathrm{D}}{ }^{19}=+63.3^{\circ}(c=1.01$, $\mathrm{CHCl}_{3}$ ); ${ }^{1} \mathrm{H}$ NMR (200 MHz, $\left.\mathrm{C}_{6} \mathrm{D}_{6}, \mathrm{C}_{6} \mathrm{H}_{6}=7.20 \mathrm{ppm}\right): \delta=7.8-7.6(\mathrm{~m}, 10 \mathrm{H}, \mathrm{Ar}), 7.3-7.0(\mathrm{~m}$, $15 \mathrm{H}, \mathrm{Ar}), 6.34(\mathrm{dd}, J=0.8,6.0 \mathrm{~Hz}, 1 \mathrm{H} 1-\mathrm{H}), 5.69$ (d, $\left.J=6.4 \mathrm{~Hz}, 1 \mathrm{H}, 1-\mathrm{H}^{\prime}\right), 5.13$ ( d, $J=3.2 \mathrm{~Hz}$, $\left.1 \mathrm{H}, 4-\mathrm{H}^{\prime}\right), 4.80$ (dd, $\left.J=3.6,6.0 \mathrm{~Hz}, 1 \mathrm{H}, 2-\mathrm{H}\right), 4.50$ (ddt, $\left.J=0.8,3.6,4.0 \mathrm{~Hz}, 1 \mathrm{H}, 3-\mathrm{H}\right), 4.24$ (dq, $J=6.0,6.4 \mathrm{~Hz}, 1 \mathrm{H}, 5-\mathrm{H}), 3.96(\mathrm{dd}, J=4.0,6.0 \mathrm{~Hz}, 1 \mathrm{H}, 4-\mathrm{H}), 3.86\left(\mathrm{~m}, 2 \mathrm{H}, 2-\mathrm{H}^{\prime}, 5-\mathrm{H}^{\prime}\right), 3.72(\mathrm{~d}$, $\left.J=3.6 \mathrm{~Hz}, 1 \mathrm{H}, 3-\mathrm{H}^{\prime}\right), 1.64(\mathrm{~d}, J=6.4 \mathrm{~Hz}, 3 \mathrm{H}, 6-\mathrm{H}), 1.21,1.14,1.08(3 \mathrm{~s}, 27 \mathrm{H}, 3 \mathrm{x} t \mathrm{Bu}), 1.0$ (d, $J=$ $\left.6.4 \mathrm{~Hz}, 3 \mathrm{H}, 6-\mathrm{H}^{\prime}\right), 0.33,0.25\left(2 \mathrm{~s}, 6 \mathrm{H}, \mathrm{Si}\left(\mathrm{CH}_{3}\right)_{2}\right) ;{ }^{13} \mathrm{C}-\mathrm{NMR}\left(100 \mathrm{MHz}, \mathrm{C}_{6} \mathrm{D}_{6}, \mathrm{C}_{6} \mathrm{H}_{6}=128.0 \mathrm{ppm}\right)$ $\delta: 136.5,136.4,136.3,136.2,131.9,130.2,130.1,130.0,129.9,129.0,128.3,128.1,126.5(\mathrm{~d}$, Ar), 133.7, 133.6, 133.5, 133.4 (s, Ar), 143.1 (d, C-1), 103.8 (d, C'-1), 103.1 (d, C-2), 79.7 (d, C4), 78.9, 78.8 (d, C'-4, $\left.\mathrm{C}^{\prime}-5\right), 73.7$ (d, C'-3), 73.3 (d, C-5), 67.1 (d, C-3), 49.5 (d, C'-2), 27.4, 27.2, 26.2 (3q, $\mathrm{CH}_{3}$ of $t \mathrm{Bu}$ ), 20.2 (q, $\left.\mathrm{C}^{\prime}-6\right), 19.3,19.2,18.3$ (3s, $t \mathrm{Bu}$ ), 17.6 (q, C-6), -4.0, -4.6 (q, $\left.\mathrm{Si}\left(\mathrm{CH}_{3}\right)_{2}\right)$; combustion analysis calcd (\%) for $\mathrm{C}_{56} \mathrm{H}_{74} \mathrm{O}_{6} \mathrm{SeSi}_{3}$ : C 66.83, H 7.41; found $\mathrm{C} 66.89, \mathrm{H}$ 7.83.

Acetyl [3',4'-bis-O-(tert-butyldiphenylmethylsilyl)-2',6'-dideoxy-2'-phenylselenyl- $\beta$-L-glucohexopyranosyl] (1-4)-3-O-tert-butyldimethylsilyl-2,6-dideoxy-2-iodo- $\beta$-L-gluco-hexopyranose 11a and acetyl $\left[3^{\prime}, 4^{\prime}\right.$-bis- $O$-(tert-butyldiphenylmethylsilyl)-2 $2^{\prime}, 6^{\prime}$-dideoxy-2'-phenylselenyl- $\beta$ L-gluco-hexopyranosyl] (1-4)-3-O-tert-butyldimethylsilyl-2,6-dideoxy-2-iodo- $\alpha$-L-mannohexopyranose (11b). A solution of disaccharide $10(215 \mathrm{mg}, 0.21 \mathrm{mmol})$ in abs. dichloromethane $(8 \mathrm{ml})$ was treated with polymer-bound reagent $4(100 \mathrm{mg})$ for $48 \mathrm{~h}$ at $\mathrm{rt}$. Filtration and removal of the solvent in vacuo gave an oil which contained two diastereomers 11a and 11b. These were separated by column chromatography (silica gel; petroleum ether / ethyl acetate $50: 1 ; 221 \mathrm{mg}, 0.185 \mathrm{mmol} ; 87 \%$ ).

${ }^{1 s t}$ fraction 11a. colorless solid; m.p.: $102^{\circ} \mathrm{C} ;[\alpha]_{\mathrm{D}}{ }^{22}=+22.9^{\circ}\left(c=0.99, \mathrm{CHCl}_{3}\right) ;{ }^{1} \mathrm{H}$ NMR $(400$ $\left.\mathrm{MHz}, \mathrm{C}_{6} \mathrm{D}_{6}\right): \delta=7.78-7.50(\mathrm{~m}, 9 \mathrm{H}, \mathrm{Ar}), 7.31-7.02(\mathrm{~m}, 16 \mathrm{H}, \mathrm{Ar}), 6.01(\mathrm{~d}, J=10.0 \mathrm{~Hz}, 1 \mathrm{H} 1-\mathrm{H})$, 5.66 (d, $\left.J=7.6 \mathrm{~Hz}, 1 \mathrm{H}, 1-\mathrm{H}^{\prime}\right), 5.20$ ( d, $\left.J=3.2 \mathrm{~Hz}, 1 \mathrm{H}, 3-\mathrm{H}^{\prime}\right), 4.04$ (dd, $J=10.0,10.0 \mathrm{~Hz}, 1 \mathrm{H}, 2-$ H), $3.99\left(\mathrm{~m}, 1 \mathrm{H}, 2-\mathrm{H}^{\prime}\right), 3.80(\mathrm{dd}, J=8.0,10.0 \mathrm{~Hz}, 1 \mathrm{H}, 3-\mathrm{H}), 3.69$ (q, $\left.J=6.8 \mathrm{~Hz}, 1 \mathrm{H}, 5^{\prime}-\mathrm{H}\right), 3.56$ (dd, $J=8.0,8.8 \mathrm{~Hz}, 1 \mathrm{H}, 4-\mathrm{H}), 3.50$ (d, $\left.J=3.2 \mathrm{~Hz}, 1 \mathrm{H}, 4-\mathrm{H}^{\prime}\right), 3.44$ (dq, $\left.J=6.48 .8 \mathrm{~Hz}, 1 \mathrm{H}, 5-\mathrm{H}\right)$, 1.90 (d, J=6.4 Hz, 3H, 6-H), 1.72 (s, 3H, OAc), 1.32, 1.20, 1.15 (3s, 27H, 3x $t \mathrm{Bu}), 0.86$ (d, J= $\left.6.8 \mathrm{~Hz}, 3 \mathrm{H}, 6-\mathrm{H}^{\prime}\right), 0.68,0.54\left(2 \mathrm{~s}, 6 \mathrm{H}, \mathrm{Si}\left(\mathrm{CH}_{3}\right)_{2}\right) ;{ }^{13} \mathrm{C}-\mathrm{NMR}\left(100 \mathrm{MHz}, \mathrm{C}_{6} \mathrm{D}_{6}\right) \delta: 168.2(\mathrm{~s}, \mathrm{OAc})$, 136.5, 136.4, 136.3, 136.2, 131.8, 130.2, 130.1, 129.9, 129.2, 128.3, 128.0, 127.9, 127.8, 126.8 (d, Ar), 133.9, 133.6, 133.3, 133.2, 133.0 (s, Ar), 103.3 (d, C'-1) 94.4 (d, C-1), 81.5 (d, C-4), 80.3 (d, C-5), 80.1 (d, C'-3), 76.9 (d, C-3), 73.6 (d, C'-4), 73.1 (d, C'-5), 49.9 (d, C'-2), 35.8 (d, C-2), 27.4, 27.2, 27.0 (3q, $\mathrm{CH}_{3}$ of $t \mathrm{Bu}$ ), 20.3 (q, OAc), 19.9 (q, C-6), 19.6 (q, C'-6), 19.3, 18.9, $18.5(3 \mathrm{~s}, t \mathrm{Bu}), 0.3,-2.4\left(2 \mathrm{q}, \mathrm{Si}\left(\mathrm{CH}_{3}\right)_{2}\right)$; combustion analysis calcd (\%) for $\mathrm{C}_{58} \mathrm{H}_{77} \mathrm{IO}_{8} \mathrm{SeSi}_{3}: \mathrm{C}$ 58.42, H 6.51; found C 58.68, H 6.43.

${ }^{2 n d}$ fraction 11b. colorless solid; m.p.: $68^{\circ} \mathrm{C} ;[\alpha]_{\mathrm{D}}{ }^{22}=+31.9^{\circ}\left(c=1.01, \mathrm{CHCl}_{3}\right) ;{ }^{1} \mathrm{H}$ NMR $(400$ $\left.\mathrm{MHz}, \mathrm{C}_{6} \mathrm{D}_{6}\right): \delta=7.82-7.60(\mathrm{~m}, 9 \mathrm{H}, \mathrm{Ar}), 7.32-7.00(\mathrm{~m}, 16 \mathrm{H}, \mathrm{Ar}), 6.74(\mathrm{~d}, J=4.0 \mathrm{~Hz}, 1 \mathrm{H} \mathrm{1-H})$, 
$5.72\left(\mathrm{~d}, J=7.2 \mathrm{~Hz}, 1 \mathrm{H}, 1-\mathrm{H}^{\prime}\right), 5.14$ ( d, $\left.J=4.0 \mathrm{~Hz}, 1 \mathrm{H}, 3-\mathrm{H}^{\prime}\right), 4.47$ (dd, $J=4.0,4.0 \mathrm{~Hz}, 1 \mathrm{H}, 2-\mathrm{H}$ ), 4.00 (m, 2H, 4-H, 5-H), 3.92 (d, J= 7.2 Hz, 1H, 2-H'), 3.90 (dq, J=4.0, 7.2 Hz, 1H, 5'-H), 3.81 (br s, 1H, 3-H), 3.71 (d, J=4.0 Hz, 1H, 4-H'), 1.82 (d, J=4.8 Hz, 3H, 6-H), 1.66 (s, 3H, OAc), 1.21, 1.19, $1.18(3 \mathrm{~s}, 27 \mathrm{H}, 3 \mathrm{x} t \mathrm{Bu}), 1.02\left(\mathrm{~d}, J=7.2 \mathrm{~Hz}, 3 \mathrm{H}, 6-\mathrm{H}^{\prime}\right), 0.47,0.32\left(2 \mathrm{~s}, 6 \mathrm{H}, \mathrm{Si}\left(\mathrm{CH}_{3}\right)_{2}\right)$; ${ }^{13} \mathrm{C}-\mathrm{NMR}\left(100 \mathrm{MHz}, \mathrm{C}_{6} \mathrm{D}_{6}\right)$ ): 168.1 (s, OAc), 136.5, 136.4, 136.3, 136.2, 131.8, 130.3, 130.1, 129.0, 127.9, 127.3, 126.5 (d, Ar), 133.7, 133.5, 133.4, 133.3, 133.0 (s, Ar), 104.8 (d, C'-1) 94.6 (d, C-1), 82.0 (d, C-5), 79.6 (d, C-5), 79.0 (d, C'-3), 73.8 (d, C'-4), 71.6 (d, C-4), 71.2 (d, C-3), 49.8 (d, C'-2), 30.2 (d, C-2), 27.3, 27.2, 26.4 (3q, $\mathrm{CH}_{3}$ of $\left.t \mathrm{Bu}\right), 20.3$ (q, OAc), 20.1 (q, C-6), 19.6 (q, $\left.\mathrm{C}^{\prime}-6\right), 19.3,19.1,18.4(3 \mathrm{~s}, \mathrm{Bu}),-3.2,-4.8\left(2 \mathrm{q}, \mathrm{Si}\left(\mathrm{CH}_{3}\right)_{2}\right)$; combustion analysis calcd $(\%)$ for $\mathrm{C}_{58} \mathrm{H}_{77} \mathrm{IO}_{8} \mathrm{SeSi}_{3}$ : C 58.42, H 6.51; found C 58.12, H 6.67.

Acetyl 4-O-benzoyl-3-O-tert-butyldimethylsilyl-2,6-dideoxy-2-iodo- $\alpha$-D-manno-hexopyranose 13a and acetyl 4-O-benzoyl-3-O-tert-butyldimethylsilyl-2,6-dideoxy-2-iodo- $\beta$-D-glucohexopyranose (13b). A solution of glycal 12 (6.2 g, $17.8 \mathrm{mmol})$ in abs. dichloromethane (50 $\mathrm{ml})$ was treated with polymer-bound reagent $4(4.7 \mathrm{~g})$ for $52 \mathrm{~h}$ at rt. Filtration and removal of the solvent in vacuo gave an oil which contained two diastereomers 13a and 13b $(2.7: 1 ;>95 \%$ crude yield). These were separated by column chromatography (silica gel; petroleum ether / ethyl acetate 40:1).

$\mathbf{1}^{\text {st }}$ fraction 13a. $(5.9 \mathrm{~g}, 11.1 \mathrm{mmol} ; 62.4 \%)$; colorless crystals, $\mathrm{mp}: 90^{\circ} \mathrm{C} ;[\alpha]_{\mathrm{D}}{ }^{23.5}=-37.2^{\circ}(c=$ $\left.1.25, \mathrm{CHCl}_{3}\right) ;{ }^{1} \mathrm{H}-\mathrm{NMR}\left(200 \mathrm{MHz}, \mathrm{CDCl}_{3}, \mathrm{CHCl}_{3}=7.26 \mathrm{ppm}\right) \delta: 8.10-7.97$ (m, $2 \mathrm{H}, \mathrm{Ph}_{\text {ortho }}$ ), 7.64-7.52 (m, 1H, $\left.\mathrm{Ph}_{\text {para }}\right), 7.51-7.38\left(\mathrm{~m}, 2 \mathrm{H}, \mathrm{Ph}_{\text {meta }}\right), 6.41(\mathrm{~d}, 1 \mathrm{H}, J=1.9 \mathrm{~Hz}, 1-\mathrm{H}), 5.32$ (dd, 1H, $J=9.6,8.8 \mathrm{~Hz}, 4-\mathrm{H}), 4.31$ (dd, 1H, $J=4.3,1.9 \mathrm{~Hz}, 2-\mathrm{H}), 4.08$ (ddq, 1H, $J=9.6,6.3,0.5 \mathrm{~Hz}, 5-$ H), 3.41 (ddd, 1H, J=8.8, 4.3, 0.5 Hz, 3-H), 2.17 (s, 3H, 1-O-Ac), 1.27 (d, 3H, J=6.3 Hz, 6-H), 0.78 (s, 9H, 3-O-Si-tBu), 0.05, -0.12 (2s, 6H, 3-O-Si-Me $)$; ${ }^{13} \mathrm{C}-\mathrm{NMR}\left(50 \mathrm{MHz}, \mathrm{CDCl}_{3}, \mathrm{CDCl}_{3}=\right.$ 77.0 ppm) $\delta:$ 168.6, 165.2 (2s, O-(O)CMe, O-(O)CPh), 133.2 (d, $\left.\mathrm{Ph}_{\text {para }}\right), 129.8\left(\mathrm{~s}, \mathrm{Ph}_{\text {ipso }}\right), 129.8$, 128.4 (2d, arom. CH), 95.3 (d, C-1), 75.5, 69.9, 67.7 (3d, C-3, C-4, C-5), 34.9 (d, C-2), 25.4 (s, $\mathrm{Si}-\mathrm{BBu}), 20.9$ (q, O- $\left.(\mathrm{O}) \mathrm{CCH}_{3}\right), 17.7$ (q, Si-tBu), 17.6 (q, C-6), -4.6, -4.7 (2q, $\left.\mathrm{Si}\left(\mathrm{CH}_{3}\right)_{2}\right)$; combustion analysis calcd (\%) for $\mathrm{C}_{21} \mathrm{H}_{31} \mathrm{IO}_{6} \mathrm{Si}$ : C 47.19, $\mathrm{H}$ 5.85; found: $\mathrm{C} 47.01$; $\mathrm{H}$ 5.87.

$2^{\text {nd }}$ fraction 13b. (2.18 g, $4.1 \mathrm{mmol} ; 23.0 \%$ ); colourless crystals, mp: $118^{\circ} \mathrm{C} ;[\alpha]_{\mathrm{D}}{ }^{23.0}=+25.1^{\circ}$ $\left(c=0.28, \mathrm{CHCl}_{3}\right) ;{ }^{1} \mathrm{H}-\mathrm{NMR}\left(200 \mathrm{MHz}, \mathrm{CDCl}_{3}, \mathrm{CHCl}_{3}=7.26 \mathrm{ppm}\right) \delta: 8.14-7.96\left(\mathrm{~m}, 2 \mathrm{H}, \mathrm{Ph}_{\text {ortho }}\right)$, 7.64-7.52 (m, 1H, $\left.\mathrm{Ph}_{\text {para }}\right), 7.52-7.38\left(\mathrm{~m}, 2 \mathrm{H}, \mathrm{Ph}_{\text {meta }}\right), 5.91$ (d, 1H, J=9.5 Hz, 1-H), 5.01 (dd, 1H, $J=9.7,8.4 \mathrm{~Hz}, 4-\mathrm{H}), 4.15$ (dd, $1 \mathrm{H}, J=9.8,8.4 \mathrm{~Hz}, 3-\mathrm{H}), 3.95$ (dd, 1H, J=9.8, 9.5 Hz, 2-H), 3.73 (dq, 1H, J=9.7, 6.2 Hz, 5-H), 2.15 (s, 3H, 1-O-Ac), 1.21 (d, 3H, J=6.2 Hz, 6-H), 0.80 (s, 9H, 3O-Si-tBu), 0.22, -0.19 (2s, 6H, 3-O-Si-Me $) ;{ }^{13} \mathrm{C}-\mathrm{NMR}\left(50 \mathrm{MHz}, \mathrm{CDCl}_{3}, \mathrm{CDCl}_{3}=77.0 \mathrm{ppm}\right) \delta$ : 168.8, 165.4 (2s, O-(O)CMe, O-(O)C-Ph), 133.4 (d, $\mathrm{Ph}_{\text {para }}$ ), 129.7 (s, $\mathrm{Ph}_{\text {ipso }}$ ), 129.7, 128.5 (2d, Ph), 94.1 (d, C-1), 76.7, 76.5, 71.3 (3d, C-3, C-4, C-5), 33.7 (d, C-2), 26.0 (s, Si-tBu), 20.8 (q, O$\left.(\mathrm{O}) \mathrm{CCH}_{3}\right), 18.1$ (q, Si-tBu), 17.5 (q, C-6), -3.2, -3.5 (2q, $\left.\mathrm{Si}\left(\mathrm{CH}_{3}\right)_{2}\right)$; combustion analysis calcd (\%) for $\mathrm{C}_{21} \mathrm{H}_{31} \mathrm{IO}_{6} \mathrm{Si}$ : C 47.19, H 5.85; found: C 47.11; $\mathrm{H} 5.73$. 
Methyl 4-O-benzoyl-3-O-tert-butyldimethylsilyl-2,6-dideoxy-2-iodo- $\alpha$-D-manno-hexopyranoside (16). To a solution of glycosyl acetate 13a $(200 \mathrm{mg}, 0.374 \mathrm{mmol})$ in dry diethyl ether $(20 \mathrm{ml})$ at $70^{\circ} \mathrm{C}$ was added dry methanol $(1 \mathrm{ml})$ and polymer-bound silyl triflate 14 (150 mg, about 0.6 eq.). The reaction mixture was shaken for $18 \mathrm{~h}$ at $\mathrm{rt}$ and Amberlyst A-21 15 (250 mg) was added for neutralization. Filtration at $-40^{\circ} \mathrm{C}$ and washing of the resin with diethyl ether $(3 \times 10 \mathrm{ml})$ gave a solution which was concentrated in vacuo to yield target glycoside 16 (181 $\mathrm{mg}, 0.357 \mathrm{mmol}$; $95.4 \%)$; colorless oil; $[\alpha]_{\mathrm{D}}{ }^{23.5}=-43.6^{\circ}\left(c=1.35, \mathrm{CHCl}_{3}\right) ;{ }^{1} \mathrm{H}-\mathrm{NMR}\left(200 \mathrm{MHz}, \mathrm{CDCl}_{3}, \mathrm{CHCl}_{3}=\right.$ 7.26 ppm) $\delta: ~ 8.10-7.98\left(\mathrm{~m}, 2 \mathrm{H}, \mathrm{Ph}_{\text {ortho }}\right), 7.63-7.51$ (m, 1H, $\left.\mathrm{Ph}_{\text {para }}\right), 7.51-7.38$ (m, 2H, $\left.\mathrm{Ph}_{\text {meta }}\right), 5.26$ (dd, 1H, $J=9.5,9.0 \mathrm{~Hz}, 4-\mathrm{H}), 5.08$ (d, 1H, $J=1.2 \mathrm{~Hz}, 1-\mathrm{H}), 4.32$ (dd, 1H, J= 4.5, $1.2 \mathrm{~Hz}, 2-\mathrm{H})$, 3.96 (dq, 1H, J=9.5, $6.5 \mathrm{~Hz}, 5-\mathrm{H}), 3.45$ (dd, 1H, J=9.0, $4.5 \mathrm{~Hz}, 3-\mathrm{H}), 3.40$ (s, 3H, OMe), 1.26 (d, 3H, J=6.5 Hz, 6-H), 0.77 (s, 9H, Si-tBu), 0.05, -0.15 (2s, 6H, Si-Me $)_{2}$; ${ }^{13} \mathrm{C}-\mathrm{NMR}(50 \mathrm{MHz}$, $\left.\mathrm{CDCl}_{3}, \mathrm{CDCl}_{3}=77.0 \mathrm{ppm}\right) \delta: 165.4$ (s, CO-Ph), 133.1 (d, $\left.\mathrm{Ph}_{\text {para }}\right), 130.0\left(\mathrm{~s}, \mathrm{Ph}_{\text {ipso }}\right), 129.7,128.3$ (2d, Ph), 102.7 (d, C-1), 76.2, 67.7, 67.1 (3d, C-3, C-4, C-5), 55.2 (q, OMe), 37.2 (d, C-2), 25.5 (q, Si-tBu), 17.8 (s, Si-tBu), 17.7 (q, C-6), -4.6, -4.8 (2q, $\left.\mathrm{SiMe}_{2}\right)$; combustion analysis calcd (\%) for $\mathrm{C}_{20} \mathrm{H}_{31} \mathrm{IO}_{5} \mathrm{Si}$ : C 47.43, $\mathrm{H}$ 6.17; found: C 47.57; $\mathrm{H} 6.25$.

Methyl 4-O-benzoyl-2,6-dideoxy- $\alpha$-D-arabino-hexopyranoside (18). To a solution of methyl glycoside 16 (160 mg, $0.32 \mathrm{mmol})$ and $\mathrm{Bu}_{3} \mathrm{SnH}(0.33 \mathrm{ml}, 1.26 \mathrm{mmol}, 4$ eq.) in dry toluene (15 $\mathrm{ml})$ was added AIBN (10 mg). The solution was heated for $1 \mathrm{~h}$ at reflux. The solution was concentrated under reduced pressure and the crude product was purified by column chromatography (petroleum ether / ethyl acetate 30:1) to yield the deiodinated glycoside ( 115 $\mathrm{mg}, 0.3 \mathrm{mmol}, 95.6 \%)$.

This material $(97 \mathrm{mg}, 255 \mathrm{mmol})$ was dissolved in dichloromethane $(10 \mathrm{ml})$ and was dropwise treated with $\mathrm{HF} /$ pyridine complex $(0.1 \mathrm{ml}, 3.8 \mathrm{mmol}, 15$ eq. $)$ for $2 \mathrm{~min}$ at room temperature. To this reaction mixture was added Amberlite A-200 ( $\mathrm{Na}^{+}$-Form, $100 \mathrm{mg}$ ) and shaking was continued for $30 \mathrm{~min}$. Filtration and washing of the resin with dichloromethane $(3 \mathrm{x}$ $5 \mathrm{ml}$ ) gave a solution which was concentrated in vacuo to yield the target glycoside 18 (54 mg, $0.19 \mathrm{mmol} ; 75.5 \%) ;{ }^{1} \mathrm{H}-\mathrm{NMR}\left(200 \mathrm{MHz}, \mathrm{CDCl}_{3}, \mathrm{CHCl}_{3}=7.26 \mathrm{ppm}\right) \delta: 8.10-7.96$ (m, 2H, $\mathrm{Ph}_{\text {ortho}}$ ), 7.62-7.50 (m, 1H, $\mathrm{Ph}_{\text {para }}$ ), 7.49-7.35 (m, 2H, $\mathrm{Ph}_{\text {meta }}$ ), 4.76 (dd, 1H, J= 3.8, 1.2 Hz, 1-H), $4.76(\mathrm{dd}, 1 \mathrm{H}, J=9.7,9.1 \mathrm{~Hz}, 4-\mathrm{H}), 4.14(\mathrm{ddd}, 1 \mathrm{H}, J=11.5,9.1,5.3 \mathrm{~Hz}, 3-\mathrm{H}), 3.89$ (dq, $1 \mathrm{H}, J=$ 9.7, $6.3 \mathrm{~Hz}, 5-\mathrm{H}), 3.34$ (s, 3H, OMe), 2.23 (ddd, 1H, J=13.1, 5.3, $1.2 \mathrm{~Hz}, 2-\mathrm{H}_{\mathrm{eq}}$ ), 1.78 (ddd, 1H, $\left.J=13.1,11.5,3.8 \mathrm{~Hz}, 2-\mathrm{H}_{\mathrm{ax}}\right), 1.22(\mathrm{~d}, 3 \mathrm{H}, J=6.3 \mathrm{~Hz}, 6-\mathrm{H}) ;{ }^{13} \mathrm{C}-\mathrm{NMR}\left(50 \mathrm{MHz}, \mathrm{CDCl}_{3}, \mathrm{CDCl}_{3}=\right.$ 77.0 ppm) $\delta: 166.9$ (s, 4-O-CO-Ph), 133.3 (d, $\left.\mathrm{Ph}_{\text {para }}{ }^{\prime}\right), 129.7,128.4$ (2d, Ph), 129.5 (s, $\mathrm{Ph}_{\text {ipso }}$ ), 98.2 (d, C-1), 79.3 (d, C-4), 67.4, 65.4 (dd, C-3, C-5), 54.7 (q, OMe), 38.0 (t, C-2), 17.6 (q, C-6); combustion analysis calcd (\%) for $\mathrm{C}_{14} \mathrm{H}_{18} \mathrm{O}_{5}$ : C 63.15, $\mathrm{H} 6.81$; found: $\mathrm{C} 63.04 \mathrm{H} 6.77$.

Methyl (4'-O-benzoyl-2',6'-dideoxy- $\alpha$-D-arabino-hexopyranosyl) (1-3)-4-O-benzoyl- $\alpha$-Darabino-hexopyranosid (19). To a solution of methyl glycoside 18 (50 mg, $0.19 \mathrm{mmol})$ and glycosyl acetate 13a (160 mg, $0.30 \mathrm{mmol}, 1.6$ eq.) in dry diethyl ether $(5 \mathrm{ml})$ at $-70^{\circ} \mathrm{C}$ was added polymer-bound silyl triflate 14 (100 mg, about 0.54 eq.). The reaction mixture was shaken for $6.5 \mathrm{~h}$ at room temperature and Amberlyst A-21 $15(60 \mathrm{mg})$ was added for neutralization. Filtration and washing of the resin with diethyl ether $(3 \times 3 \mathrm{ml})$ gave a solution which was 
concentrated in vacuo. The crude oil was purified by gel filtration (silica gel; petroleum ether / ethyl acetate $15: 1$ ) to yield the disaccharide (120 mg, $0.16 \mathrm{mmol} ; 85.6 \%$ ); ${ }^{1} \mathrm{H}-\mathrm{NMR}$ (400 MHz, $\left.\mathrm{CDCl}_{3}, \mathrm{CHCl}_{3}=7.26 \mathrm{ppm}\right) \delta:$ 8.07-7.99 (m, $\left.4 \mathrm{H}, \mathrm{Ph}_{\text {ortho }}\right), 7.63-7.52$ (m, $\left.2 \mathrm{H}, \mathrm{Ph}_{\text {para }}\right), 7.51-7.38$ (m, $4 \mathrm{H}, \mathrm{Ph}_{\text {meta }}$ ), 5.24 (br s, $\left.1 \mathrm{H}, 1-\mathrm{H}\right), 5.17$ (dd, $\left.1 \mathrm{H}, J=9.8,8.9 \mathrm{~Hz}, 4-\mathrm{H}\right), 5.01$ (dd, $1 \mathrm{H}, J=9.8,9.4 \mathrm{~Hz}$, $\left.4^{\prime}-\mathrm{H}\right), 4.80$ (d, 1H, $\left.J=3.4 \mathrm{~Hz}, 1^{\prime}-\mathrm{H}\right), 4.16$ (ddd, $\left.1 \mathrm{H}, J=11.7,9.4,5.1 \mathrm{~Hz}, 3^{\prime}-\mathrm{H}\right), 4.01$ (dq, 1H, $J=$ 9.8, $6.4 \mathrm{~Hz}, 5-\mathrm{H}), 3.94$ (dq, $1 \mathrm{H}, J=9.8,6.4 \mathrm{~Hz}, 5^{\prime}-\mathrm{H}$ ), 3.90 (dd, $\left.1 \mathrm{H}, J=4.2,1.2 \mathrm{~Hz}, 2-\mathrm{H}\right), 3.36$ (s, $3 \mathrm{H}, \mathrm{OMe}), 3.31$ (dd, $1 \mathrm{H}, J=8.9,4.2 \mathrm{~Hz}, 3-\mathrm{H}), 2.36$ (ddd, $1 \mathrm{H}, J=12.8,5.1,0.7 \mathrm{~Hz}, 2^{\prime}-\mathrm{H}_{\text {eq }}$ ), 1.92 (ddd, $1 \mathrm{H}, J=12.8,11.7,3.4 \mathrm{~Hz}, 2^{\prime}-\mathrm{H}_{\mathrm{ax}}$ ), 1.25 (d, 3H, $\left.J=6.4 \mathrm{~Hz}, 6^{\prime}-\mathrm{H}\right), 1.20$ (d, 3H, $J=6.4 \mathrm{~Hz}, 6-$ H), 0.66 (s, 9H, Si-tBu'), $-0.17,-0.22\left(2 \mathrm{~s}, 6 \mathrm{H}, \mathrm{Si}^{-\mathrm{Me}_{2}}{ }^{\prime}\right) ;{ }^{13} \mathrm{C}-\mathrm{NMR}\left(100 \mathrm{MHz}, \mathrm{CDCl}_{3}, \mathrm{CDCl}_{3}=\right.$ 77.0 ppm) $\delta:$ 165.8, 165.3 (2s, 4-O-CO-Ph, 4'-O-CO-Ph), 133.4, 133.0 (2d, $\left.\mathrm{Ph}_{\text {para }}{ }^{\prime}\right), 129.9,129.4$ (2s, $\left.\mathrm{Ph}_{\text {ipso }}{ }^{\prime}\right), 129.7,129.7$ (2d, $\left.\mathrm{Ph}_{\text {ortho }}{ }^{\prime}\right), 128.6,128.3$ (2d, $\left.\mathrm{Ph}_{\text {meta }}{ }^{\prime}\right), 103.4$ (d, C-1), 98.1 (d, C-1'), 76.9 (d, C-4), 76.5 (d, C-3'), 76.1 (d, C-4'), 67.6 (d, C-5), 67.4 (d, C-3), 65.7 (d, C-5'), 54.7 (q, OMe), 37.2 (d, C-2'), 37.0 (t, C-2'), 25.4 (q, Si-tBu'), 17.6 (q, C-6'), 17.6 (s, Si-tBu'), 17.5 (q, C-6), -5.0 (q, Si-Me ${ }^{\prime}$ ).

This material $(72 \mathrm{mg}, 97 \mu \mathrm{mol})$ and $\mathrm{Bu}_{3} \mathrm{SnH}(0.08 \mathrm{ml}, 0.3 \mathrm{mmol}, 3.1 \mathrm{eq}$.$) in dry toluene (10$ $\mathrm{ml}$ ) was added AIBN (10 mg). The solution was heated for $5 \mathrm{~h}$ at reflux. The solution was concentrated under reduced pressure and the crude product was purified by column chromatography (petroleum ether / ethyl acetate 50:1) to yield the deiodinated glycoside (47 $\mathrm{mg}$, $76 \mu \mathrm{mol}, 78.6 \%$ ). ${ }^{1} \mathrm{H}-\mathrm{NMR}\left(400 \mathrm{MHz}, \mathrm{CDCl}_{3}, \mathrm{CHCl}_{3}=7.26 \mathrm{ppm}\right) \delta: 8.08-7.99$ (m, $4 \mathrm{H}, \mathrm{Ph}_{\text {ortho }}$ ), 7.62-7.51 (m, $2 \mathrm{H}, \mathrm{Ph}_{\text {para }}$ ), 7.49-7.39 (m, $4 \mathrm{H}, \mathrm{Ph}_{\text {meta }}$ ), 5.00 (dd, $\left.1 \mathrm{H}, J=9.9,9.4 \mathrm{~Hz}, 4^{\prime}-\mathrm{H}\right), 4.92$ (d, $1 \mathrm{H}, J=3.2 \mathrm{~Hz}, 1-\mathrm{H}), 4.81$ (dd, 1H, $J=9.7,9.2 \mathrm{~Hz}, 4-\mathrm{H}), 4.80$ (d, 1H, $\left.J=3.4 \mathrm{~Hz}, 1^{\prime}-\mathrm{H}\right), 4.12$ (ddd, 1H, $J=11.6,9.4,5.2 \mathrm{~Hz}, 3^{\prime}-\mathrm{H}$ ), 4.09 (ddd, $\left.1 \mathrm{H}, J=11.3,9.2,5.1 \mathrm{~Hz}, 3-\mathrm{H}\right), 3.94$ (dq, $1 \mathrm{H}, J=$ 9.7, $6.4 \mathrm{~Hz}, 5-\mathrm{H}$ ), 3.92 (dq, $1 \mathrm{H}, J=9.9,6.3 \mathrm{~Hz}, 5^{\prime}-\mathrm{H}$ ), 3.36 (s, 3H, OMe), 2.33 (dd, $1 \mathrm{H}, J=12.9$, $\left.5.2 \mathrm{~Hz}, 2^{\prime}-\mathrm{H}_{\mathrm{eq}}\right), 1.92\left(\mathrm{ddd}, 1 \mathrm{H}, J=12.9,11.6,3.4 \mathrm{~Hz}, 2^{\prime}-\mathrm{H}_{\mathrm{ax}}\right), 1.72(\mathrm{dd}, 1 \mathrm{H}, J=13.0,5.1 \mathrm{~Hz}, 2-$ $\mathrm{H}_{\mathrm{eq}}$ ), 1.55 (ddd, $\left.1 \mathrm{H}, J=13.0,11.3,3.2 \mathrm{~Hz}, 2-\mathrm{H}_{\mathrm{ax}}\right), 1.24$ (d, 3H, $\left.J=6.3 \mathrm{~Hz}, 6^{\prime}-\mathrm{H}\right), 1.14$ (d, 3H, $J$ $=6.4 \mathrm{~Hz}, 6-\mathrm{H}), 0.63\left(\mathrm{~s}, 9 \mathrm{H}, \mathrm{Si}-t \mathrm{Bu}{ }^{\prime}\right),-0.18,-0.22\left(2 \mathrm{~s}, 6 \mathrm{H}, \mathrm{Si}_{-} \mathrm{Me}_{2}{ }^{\prime}\right) ;{ }^{13} \mathrm{C}-\mathrm{NMR}\left(50 \mathrm{MHz}, \mathrm{CDCl}_{3}\right.$, $\mathrm{CDCl}_{3}=77.0$ ppm) $\delta: 165.6$ (s, 4-O-CO-Ph, 4'-O-CO-Ph), 133.2, 132.9 (2d, $\left.\mathrm{Ph}_{\text {para }}\right), 130.1,129.8$ (2s, $\left.\mathrm{Ph}_{\text {ipso }}\right), 129.6,129.5$ (2d, $\left.\mathrm{Ph}_{\text {ortho }}\right), 128.5,128.2$ (2d, $\left.\mathrm{Ph}_{\text {meta }}\right), 99.5$ (d, C-1), 98.2 (d, C-1'), 79.3 (d, C-4), 76.8, 75.5 (2d, C-3', C-4'), 67.2 (d, C-3), 66.4, 65.8 (2d, C-5, C-5'), 54.7 (q, OMe), 39.2 (t, C-2), 37.2 (t, C-2'), 25.4 (q, Si-tBu'), 17.7, 17.5 (2q, C-6, C-6'), 17.5 (s, Si-tBu'), -4.9, -5.1 $\left(2 \mathrm{q}, \mathrm{Si}-\mathrm{Me}_{2}{ }^{\prime}\right)$.

This material $(47 \mathrm{mg}, 76 \mu \mathrm{mol})$ was dissolved in dichloromethane $(10 \mathrm{ml})$ and was dropwise treated with $\mathrm{HF} /$ pyridine complex $(0.05 \mathrm{ml}, 1.9 \mathrm{mmol}, 25$ eq. $)$ for $15 \mathrm{~min}$ at room temperature. To this reaction mixture was added Amberlite A-200 ( $\mathrm{Na}^{+}$-Form, $\left.50 \mathrm{mg}\right)$ and shaking was continued for $30 \mathrm{~min}$. Filtration and washing of the resin with dichloromethane ( $3 \times 2 \mathrm{ml})$ gave a solution which was concentrated in vacuo to yield the target glycoside 19 (22 $\mathrm{mg}, 44 \mu \mathrm{mol}$; 57.7\%); ${ }^{1} \mathrm{H}-\mathrm{NMR}\left(400 \mathrm{MHz}, \mathrm{CDCl}_{3}, \mathrm{CHCl}_{3}=7.26 \mathrm{ppm}\right) \delta: 8.09-8.00$ (m, $4 \mathrm{H}, \mathrm{Ph}_{\text {ortho }}$ ), 7.62-7.54 (m, 2H, Ph para $_{\text {) }}$ 7.50-7.41 (m, 4H, Ph meta $_{\text {) }}, 4.98$ (dd, $\left.1 \mathrm{H}, J=9.9,9.3 \mathrm{~Hz}, 4^{\prime}-\mathrm{H}\right), 4.97$ (d, 1H, J=3.8 $\mathrm{Hz}, 1-\mathrm{H}), 4.80$ (d, 1H, J=3.4 Hz, 1'-H), 4.69 (dd, 1H, $J=9.6,9.4 \mathrm{~Hz}, 4-\mathrm{H}), 4.16$ (ddd, 1H, $J=$ 11.6, 9.3, 5.2 Hz, 3'-H), 4.09 (br m, 1H, 3-H), 3.96 (dq, 1H, J= 9.6, $6.3 \mathrm{~Hz}, 5-\mathrm{H}), 3.93$ (dq, 1H, 
$\left.J=9.9,6.4 \mathrm{~Hz}, 5^{\prime}-\mathrm{H}\right), 3.37$ (s, 3H, OMe), $2.28\left(\mathrm{dd}, 1 \mathrm{H}, J=13.0,5.2 \mathrm{~Hz}, 2^{\prime}-\mathrm{H}_{\mathrm{eq}}\right), 2.22$ (br s, 1H, 3-OH), 1.93 (dd, 1H, $J=12.8,5.3 \mathrm{~Hz}, 2-\mathrm{H}_{\mathrm{eq}}$ ), 1.92 (ddd, $1 \mathrm{H}, J=13.0,11.6,3.4 \mathrm{~Hz}, 2^{\prime}-\mathrm{H}_{\mathrm{ax}}$ ), 1.55 (ddd, $\left.1 \mathrm{H}, J=12.8,11.8,3.8 \mathrm{~Hz}, 2-\mathrm{H}_{\mathrm{ax}}\right), 1.23\left(\mathrm{~d}, 3 \mathrm{H}, J=6.4 \mathrm{~Hz}, 6^{\prime}-\mathrm{H}\right), 1.20$ (d, 3H, J=6.3 Hz, 6$\mathrm{H}) ;{ }^{13} \mathrm{C}-\mathrm{NMR}\left(100 \mathrm{MHz}, \mathrm{CDCl}_{3}, \mathrm{CDCl}_{3}=77.0 \mathrm{ppm}\right) \delta: 166.9,165.7$ (2s, 4-O-CO-Ph, 4'-O-CO$\mathrm{Ph}), 133.4,133.3$ (2d, $\left.\mathrm{Ph}_{\text {para }}\right), 129.8,129.6$ (2d, $\left.\mathrm{Ph}_{\text {ortho }}\right), 129.7,129.5$ (2s, $\left.\mathrm{Ph}_{\text {ipso }}\right), 128.6,128.5$ (2d, Ph meta $_{\text {), }} 99.1$ (d, C-1), 98.2 (d, C-1'), 79.3 (d, C-4), 77.0 (d, C-4'), 74.8 (d, C-3'), 67.2 (d, C3), 65.9 (d, C-5), 65.9 (d, C-5'), 54.7 (q, OMe), 38.3 (t, C-2), 37.2 (t, C-2'), 17.7, 17.6 (2q, C-6, C-6').

Reaction of decarestrictine D 20 with polymer-bound reagent (5). Decarestrictine D 20 (5mg, $23 \mu \mathrm{mol})$ was dissolved in dry dichloromethane $(1 \mathrm{ml})$ at $\mathrm{rt}$. Then, polymer-bound iodate(I) complex 5 (74.6 mg) was added and the suspension was shaken for $2 \mathrm{~h}$ under the exclusion of light. The polymer was filtered off and washed with methanol. The solvent was removed under reduced pressure. The crude product $(11 \mathrm{mg})$ was purified by gradient column chromatography over silica gel (dichloromethane : methanol $=20: 1$ to $1: 1$ ).

$1^{\text {st }}$ fraction 21a and 21b. ( 1:0.36; $\left.5 \mathrm{mg}, 13.9 \mu \mathrm{mol}, 60 \%\right)$ : colourless oil; first isomer 21a: ${ }^{1} \mathrm{H}$ NMR (400 MHz, $\left.\mathrm{CDCl}_{3}\right) \delta: 5.12(\mathrm{~d}, J=7.0 \mathrm{~Hz}, 1 \mathrm{H}, 3-\mathrm{OH}), 4.80$ (dddd, $J=9.0,8.0,7.0,6.0 \mathrm{~Hz}$, $1 \mathrm{H}, 3-\mathrm{H}), 4.11(\mathrm{dd}, J=10.4,3.6 \mathrm{~Hz}, 1 \mathrm{H}, 6-\mathrm{H}), 4.07$ (dd, $J=10.4,1.2 \mathrm{~Hz}, 1 \mathrm{H}, 5-\mathrm{H}), 3.91-3.88$ (m, 1H, 7-H), 3.77, (ddq, $J=11.2,6.2,1.8 \mathrm{~Hz}, 1 \mathrm{H}, 9-\mathrm{H}), 3.52$ (d, $J=9.0,1.2 \mathrm{~Hz}, 1 \mathrm{H}, 4-\mathrm{H}), 2.83$ (dd, $\left.J=18.0,8.0 \mathrm{~Hz}, 1 \mathrm{H}, 2-\mathrm{H}_{\mathrm{eq}}\right), 2.62\left(\mathrm{dd}, J=18.0,6.0 \mathrm{~Hz}, 1 \mathrm{H}, 2-\mathrm{H}_{\mathrm{ax}}\right), 2.44$ (brs, 1H, 7-OH), 2.08 $\left(\mathrm{ddd}, J=13.2,4.6,1.8 \mathrm{~Hz}, 1 \mathrm{H}, 8-\mathrm{H}_{\mathrm{eq}}\right), 1.50\left(\mathrm{ddd}, J=13.2,11.2,11.2 \mathrm{~Hz}, 1 \mathrm{H}, 8-\mathrm{H}_{\mathrm{ax}}\right), 1.28(\mathrm{~d}, J=$ 6.2 Hz, 3H, 10-H); ${ }^{13} \mathrm{C}$ NMR (100 MHz, $\left.\mathrm{CDCl}_{3}\right) \delta: 174.2$ (q, C-1), 79.4 (t, C-6), 78.7 (t, C-4), 73.8 (t, C-7), 72.9 (t, C-9), 69.8 (t, C-3), 41.6 (s, C-8), 38.3 (t, C-5), 38.4 (s, C-2), 21.3 (p, C10).

Second isomer 21b. ${ }^{1} \mathrm{H} \mathrm{NMR}\left(400 \mathrm{MHz} \mathrm{CDCl}_{3}\right) \delta: 4.66(\mathrm{ddd}, J=5.8,5.8,4.6 \mathrm{~Hz}, 1 \mathrm{H}, 3-\mathrm{H})$, 4.30 (ddd, $J=9.0,5.8,0.8 \mathrm{~Hz}, 1 \mathrm{H}, 4-\mathrm{H}$ ), 4.08 (brd, $J=7.0 \mathrm{~Hz}, 1 \mathrm{H}, 6-\mathrm{H}$ ), 3.91 (ddd, $J=11.2$, 4.6, $1.2 \mathrm{~Hz}, 1 \mathrm{H}, 7-\mathrm{H}), 3.60$, (ddq, $J=11.2,6.2,1.8 \mathrm{~Hz}, 1 \mathrm{H}, 9-\mathrm{H}), 3.52$ (dd, $J=16.4, J=6.0 \mathrm{~Hz}, 1 \mathrm{H}, 2-$ $\mathrm{H}_{\mathrm{eq}}$ ), $3.38\left(\mathrm{dd}, J=16.4,4.4 \mathrm{~Hz}, 1 \mathrm{H}, 2-\mathrm{H}_{\mathrm{ax}}\right), 2.49$ (brd, $J=9.0 \mathrm{~Hz}$ ), 2.44 (brs, 1H, 7-OH), 2.08 $\left(\mathrm{ddd}, J=13.2,4.6,1.8 \mathrm{~Hz}, 1 \mathrm{H}, 8-\mathrm{H}_{\mathrm{eq}}\right), 1.44\left(\mathrm{ddd}, J=13.2,11.2,11.2 \mathrm{~Hz}, 1 \mathrm{H}, 8-\mathrm{H}_{\mathrm{ax}}\right), 1.25(\mathrm{~d}, J=$ $6.2 \mathrm{~Hz}, 3 \mathrm{H}, 10-\mathrm{H})$.

$2^{\text {nd }}$ fraction 22. (2.4 mg, $6.7 \mathrm{mmol} ; 29 \%$ ): light yellow oil; ${ }^{1} \mathrm{H} \mathrm{NMR}$ (400 $\mathrm{MHz}, \mathrm{CD}_{3} \mathrm{OD}$, $\mathrm{CH}_{3} \mathrm{OH}=3.31 \mathrm{ppm}$ ) $\delta: 4.06-3.98(\mathrm{~m}, 2 \mathrm{H}, 3-\mathrm{H}$ and $4-\mathrm{H}), 4.03(\mathrm{dd}, J=10.6,10.6,1 \mathrm{H}, 6-\mathrm{H}), 3.73$ (brddd, $J=10.6,10.6,4.8 \mathrm{~Hz}, 1 \mathrm{H}, 7-\mathrm{H}), 3.62$ (dd, $J=10.6,1.2 \mathrm{~Hz}, 1 \mathrm{H}, 5-\mathrm{H}), 3.54$ (ddq, $J=6.0$, 10.6, 10.6 Hz, 9-H), 2.42 (dd, $J=15.2,3.6 \mathrm{~Hz}, 1 \mathrm{H}, 2-\mathrm{H}_{\mathrm{eq}}$ ), 2.28 (dd, $J=15.2,8.4 \mathrm{~Hz}, 1 \mathrm{H}, 2-\mathrm{H}_{\mathrm{ax}}$ ), $1.88\left(\mathrm{dd},{ }^{2} J=12.8,4.8,1.6 \mathrm{~Hz}, 1 \mathrm{H}, 8-\mathrm{H}_{\mathrm{eq}}\right), 1.25\left(\mathrm{ddd}, J=12.8,10.6 .10 .6 \mathrm{~Hz}, 1 \mathrm{H}, 8-\mathrm{H}_{\mathrm{ax}}\right), 1.11(\mathrm{~d}$, $J=6.0 \mathrm{~Hz}, 10-\mathrm{H}) ;{ }^{13} \mathrm{C} \mathrm{NMR}\left(100 \mathrm{MHz}, \mathrm{CD}_{3} \mathrm{OD}, \mathrm{CD}_{3} \mathrm{OD}=39.0 \mathrm{ppm}\right) \delta: 177.3$ (q, C-1), 82.1 (t, C-5), 75.2 (t, C-4), 75.1 (t, C-7), 73.6 (t, C-3), 73.2 (t, C-9), 44.9 (s, C-8), 40.2 (s, C-2), 39.9 (t, C-6), 21.4 (p, C-10); LR-MS: (ESI) $383\left(\mathrm{M}+\mathrm{Na}^{+}\right)^{+}$. 
Successive treatment of decarestrictine D 20 with polymer-bound reagents 5 and 23: Preparation of $4 R$-(2'S, 3'S-epoxy-5'-methyl-1'R-pyranosyl)-3S-hydroxy-butanolide 24 and 4-(4S-hydroxy-6S-methyl-5,6-dihydro-4H-pyran-2-yl)-4-oxo-butanoic acid methylester (25). Decarestrictine D (1) $(39.9 \mathrm{mg}, 185 \mu \mathrm{mol})$ was dissolved in dry dichloromethane $(2 \mathrm{~mL})$ under nitrogen. Polymer-bound iodate(I) complex 5 (550 mg) was added to the solution. The resulting suspension was shaken $(300 \mathrm{rpm}$ ) After $30 \mathrm{~min}$, the suspension had turned to red, the polymer was filtered off and washed with dry dichloromethane. The solvent was removed in vacuo and the crude product $(60 \mathrm{mg})$ was dissolved in dry methanol and IRA-900(300 $\mathrm{mg}$, hydroxide form) was added. The suspension was shaken $(300 \mathrm{rpm})$ for three hours at room temperature. The polymer was filtered and washed with methanol. After removal of the solvent in vacuo the crude product was first purified by column chromatography on silica (dichloromethane : methanol $=30: 1)$. Among other fractions pure $\gamma$-lactone $24(3.6 \mathrm{mg}, 16.8$ $\mu \mathrm{mol} ; 9 \%$ ) was isolated. Then the eluent was switched (chloroform : methanol $=40: 1$ and finally toluene : ethylacetate $=2: 1)$ and a second pure compound $25(2.5 \mathrm{mg}, \mathrm{mmol} ; 6 \%)$ was isolated.

$1^{\text {st }}$ fraction 24. colourless crystals, mp.: $158^{\circ} \mathrm{C} ;[\alpha]_{\mathrm{D}}{ }^{22}=-26.3^{\circ}\left(c=0.3, \mathrm{CDCl}_{3}\right) ;{ }^{1} \mathrm{H}$ NMR $(400$ $\mathrm{MHz}, \mathrm{CDCl}_{3}$ ) $\delta: 4.8$ (dddd, $\left.J=5.4,4.4,3.8,2.0 \mathrm{~Hz}, 1 \mathrm{H}, 3-\mathrm{H}\right), 4.6(\mathrm{dd}, J=6.8,3.8,1 \mathrm{H}, 4-\mathrm{H}), 4.3$ (brd, $J=6.8 \mathrm{~Hz}, 1 \mathrm{H}, 5-\mathrm{H}), 3.5$ (ddd, $J=11.2,6.0,4.0 \mathrm{~Hz}, 1 \mathrm{H}, 9-\mathrm{H}), 3.41$ (ddd, $J=5.6,4.4 \mathrm{~Hz}$, 1H, 7-H), 3.27 (dd, $J=1.5,4.4 \mathrm{~Hz}, 1 \mathrm{H}, 6-\mathrm{H}), 3.06$ (d, J=4.4 Hz, 1H, 3-OH), 2.79 (dd, J= 17.4, $\left.5.4 \mathrm{~Hz}, 1 \mathrm{H}, 2-\mathrm{H}_{\mathrm{eq}}\right), 2.60\left(\mathrm{dd}, J=17.4,2.0 \mathrm{~Hz}, 1 \mathrm{H}, 2-\mathrm{H}_{\mathrm{ax}}\right), 1.95$ (ddd, $J=15.4,5.6,4.0,1 \mathrm{H}, 8$ - ${ }_{\text {-eq }}$ ), $1.76\left(\mathrm{dd}, J=15.4,11.2 \mathrm{~Hz}, 1 \mathrm{H}, 8-\mathrm{H}_{\mathrm{ax}}\right), 1.18(\mathrm{~d}, J=6.0 \mathrm{~Hz}, 3 \mathrm{H}, 10-\mathrm{H}) ;{ }^{13} \mathrm{C} \mathrm{NMR}(100 \mathrm{MHz}$,

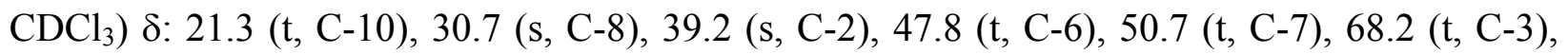
70.3 (t, C-9), 72.7 (t, C-5), 83.0 (t, C-4), 174.9 (q, C-1).

$2^{\text {nd }}$ fraction 25. colourless oil; $[\alpha]_{\mathrm{D}}{ }^{22}=7.6^{\circ}\left(c=0.25, \mathrm{CDCl}_{3}\right) ;{ }^{1} \mathrm{H}$ NMR $\left(400 \mathrm{MHz}, \mathrm{CDCl}_{3}\right) \delta$ : $5.6(\mathrm{dd}, J=2.2,2.2 \mathrm{~Hz}, 1 \mathrm{H}, 6-\mathrm{H}), 4.6$ (brddd, $J=9.0,6.8,2.2 \mathrm{~Hz}, 1 \mathrm{H}, 7-\mathrm{H}), 4.15$ (ddq, $J=11.4$, 6.4, $1.6 \mathrm{~Hz}, 1 \mathrm{H}, 9-\mathrm{H}), 3.6\left(\mathrm{~s}, 1 \mathrm{H}, \mathrm{OCH}_{3}\right), 2.98\left(\mathrm{t}, J=6.8 \mathrm{~Hz}, 1 \mathrm{H}, 3-\mathrm{H}_{\alpha}\right), 2.97$ (t, J=6.8 Hz, 1H, 3$\left.\mathrm{H}_{\beta}\right), 2.61(\mathrm{t}, J=6.8 \mathrm{~Hz}, 2 \mathrm{H}, 2-\mathrm{H}), 2.2\left(\mathrm{ddd}, J=13.2,6.8,1.6 \mathrm{~Hz}, 1 \mathrm{H}, 8-\mathrm{H}_{\mathrm{eq}}\right), 1.6$ (ddd, $J=13.2$, $\left.11.4,9.8 \mathrm{~Hz}, 1 \mathrm{H}, 8-\mathrm{H}_{\mathrm{ax}}\right), 1.4(\mathrm{~d}, J=6.4 \mathrm{~Hz}, 3 \mathrm{H}, 10-\mathrm{H}) ;{ }^{13} \mathrm{C} \mathrm{NMR}\left(100 \mathrm{MHz}, \mathrm{CDCl}_{3}\right) \delta: 19.8(\mathrm{p}$, C-10), 26.4 (s, C-2), 31.5 (t, C-3), 37.5 (s, C-8), 50.8 (p, $\left.\mathrm{OCH}_{3}\right), 62.6$ (t, C-7), 71.3 (t, C-9), 109.3 (t, C-6), 149.8 (t, C-5), 172.3 (q, C-1), 194.3 (q, C-4).

$\mathrm{X}$-ray crystallographic study of 24 . Crystal data are given in Table 2 . A single crystal $(0.63 \mathrm{x}$ $0.48 \times 0.30 \mathrm{~mm}$ ) of 24 was investigated on a Stoe IPDS area detector diffractometer using

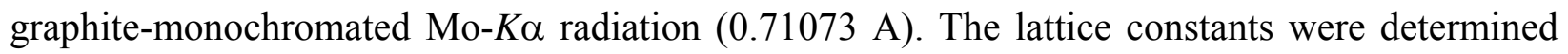
by the automatic Search and Indexing routines of the diffractometer. Indexing and refinement of 5271 reflections in the range $6.3^{\circ}<2 \theta<55.3^{\circ}$ led to a monoclinic unit cell with the dimensions $\mathrm{a}=6.454(2) \mathrm{A}, \alpha=90^{\circ}, \mathrm{b}=8.509$ (2) A, $\beta=108.41(3)^{\circ}, \mathrm{c}=10.032(3) \mathrm{A}, \gamma=90^{\circ}(\mathrm{V}=522.7(3)$ $\left.\mathrm{A}^{3}, \mathrm{Z}=2,1.361 \mathrm{Mg} / \mathrm{m}^{3}\right)$. The space group $\mathrm{P} 2{ }_{1}{ }^{29}$ was determined by the reflection conditions. The intensity data of 9205 reflections were measured at room temperature $(300(2) \mathrm{K})$ in the range of $3.21^{\circ}<-\theta<-28.24^{\circ}$. For Lp correction and data processing the programs of the Stoe IPDS software package were used. The structure was solved by direct methods using the program 
SHELXS- $86{ }^{30}$ The atomic parameters of the molecule were then completed by Fourier differences syntheses and refined (anisotropic displacement parameters for non $\mathrm{H}$-atoms) by full-matrix least squares $\left(\mathrm{F}^{2}\right)$ to the attainable $R$ values $R 1=0.0310$ [for 1757 reflections $\mathrm{F}_{\mathrm{o}}>$ $4 \sigma(\mathrm{Fo})], w R 2=0.0661$ (for all 2468 unique reflections). The hydrogen atoms were included as riding atoms. Crystallographic data (excluding structure factors) for the structure reported in this paper have been deposited with the Cambridge Crystallographic Data Center. Copies of the data can be obtained free of charge on application to The Director, CCDC, 12 Union Road, Cambridge CB2 1EZ, UK [Fax (intenational) +44(0)1223 336-033; email: deposit@chemcrys.cam.ac.uk].

\section{Acknowledgements}

This work was supported by the Fonds der Chemischen Industrie. We are grateful to Solvay Pharmaceuticals (Hannover) and Bayer AG (Leverkusen) for the donation of chemicals. We thank R. Thiericke (CyBio, Jena) for a sample of decarestrictine $2 \mathbf{2 0}$.

\section{References and Notes}

1. (a) Gladysz, J. A.; Boone, J. Angew. Chem. 1997, 109, 567; Angew. Chem., Int. Ed. 1997, 36, 551. (b) Rodriguez, J.; Dulcère, J.-P. Synthesis 1993, 1177.

2. For excellent overviews on the properties and chemistry of hypervalent iodine(III) reagents please refer to: (a) Varvoglis, A. The Organic Chemistry of Polycoordinated Iodine; VCH Verlagsgesellschaft mbH: Weinheim, 1992. (b) Varvoglis, A., Hypervalent Iodine In Organic Synthesis; Academic Press: San Diego, London; 1997.

3. The first report on this oxidation was disclosed by: (a) Doleschall, G.; Tóth, G. Tetrahedron 1980, 36, 1649. (b) Szántay, C.; Blaskó, G.; Mbárczai-Beke; Péchy, P.; Dörney, G. Tetrahedron Lett. 1980, 21, 3509.

4. Synthetic applications were reported by: (a) de Armas, P.; Concepción, J. I.; Francisco, C. G.; Hernández, R.; Salazar, J. A.; Suárez, E. J. Chem. Soc., Perkin Trans. I 1989, 405. (b) Matsuo, K.; Ishida, S.; Takuno, Y. Chem. Pharm. Bull. 1994, 42, 1149.

5. Scheffler, J. E.; Story, L. J. J. Am. Chem. Soc. 1967, 89, 2333.

6. Hadjiarapoglou, L.; Spyroudis, S.; Varvoglis, A. J. Chem. Res. (S) 1983, 50.

7. Sourkouni-Argirusi, G.; Kirschning, A. Org. Lett. 2000, 2, 3781.

8. Monenschein, H.; Sourkouni, G.; Schubothe, K. M.; O’Hare, T.; Kirschning, A. Org. Lett. 1999, 1, 2101.

9. Kirschning, A.; Monenschein, H.; Schmeck, C. Angew. Chem. 1999, 111, 2720.

10. Jaunzems, J.; Hofer, E.; Jesberger, M.; Sourkouni-Argirusi, G.; Kirschning, A. Angew. Chem., Int. Ed.; 2003 accepted for publication. 
11. (a) Kirschning, A.; Bechthold, A.; Rohr, J. Top. Curr. Chem. 1997, 188, 1. (b) Kennedy, J. F., White, C. A. Bioactive Carbohydrates in Chemistry, Biochemistry, and Biology; Ellis Horwood: Chichester, 1983. (c) Danishefsky, S. J.; Bilodeau, M. T. Angew.Chem. 1996, 108, 1482; Angew. Chem., Int. Ed. 1996, 35, 1380.

12. (a) Jesberger, M.; Jaunzems, J.; Jung, A.; Jas, G.; Schönberger, A.; Kirschning, A. Synlett 2000, 1289. (b) Hunt, J. A.; Roush, W. R.; J. Am. Chem. Soc. 1996, 118, 9998.

13. Reviews: (a) Polymers as Aids in Organic Chemistry Mathur, N. K. Narang, C. K.; Williams, R. E. Ed; Academic Press 1980, p 105. (b) Kahne, D., Curr. Opin. Chem. Biol. 1997 1, 130. (c) Danishefsky, S. J.; Roberge, J. Y. Glycopept. Relat. Compd. 1997, p 245. (d) Sofia, M. J. Mol. Div. 1998, 3, 75. (e) Schweizer, F.; Hindsgaul, O. Curr. Opin. Chem. Biol. 1999, 3, 291. (f) Osborn, H. M. I.; Khan, T. H. Tetrahedron 1999, 55, 1807. (g) St. Hilaire, P. M.; Meldal, M. Angew. Chem. 2000, 112, 1210; Angew. Chem., Int. Ed. 2000, 39, 1162.

14. Recent reviews on polymer-supported reagents: (a) Kirschning, A.; Monenschein, H.; Wittenberg, R. Angew. Chem., Int. Ed. 2001, 40, 650. (b) Ley, S. V.; Baxendale, I. R.; Bream, R. N.; Jackson, P. S.; Leach, A. G.; Longbottom, D. A.; Nesi, M.; Scott, J. S.; Storer, R. I.; Taylor, S. J. J. Chem. Soc., Perkin Trans. I 2000, 3815. (c) Drewry, D. H.; Coe, D. M.; Poon, S. Med. Res. Rev. 1999, 19, 97.

15. Kirschning, A.; Jesberger, M.; Monenschein, H. Tetrahedron Lett. 1999, 40, 8999.

16. (a) Krohn, K.; Rohr, J. Top. Curr. Chem. 1997, 188, 127. b) Rohr, J.; Thiericke, R. Nat. Prod. Rep. 1992, 9, 103.

17. Beau, J.-M.; Perez, M. Tetrahedron Lett. 1989, 30, 75.

18. (a) Roush, W. R.; Briner, K.; Sebesta, D. P. Synlett 1993, 264. (b) Kosma, P.; Sekljic, H.; Balint, G. J. Carbohydr. Chem. 1996, 15, 701. (c) Lafont, D.; Boullanger, P.; Carvalho, F.; Vottero, P. Carbohydr. Res. 1997, 297, 117. (d) Lafont, D.; Boullanger, P.; Rosenzweig, M. J. Carbohydr. Chem. 1998, 17, 1377. (e) Kirschning, A.; Plumeier, C., Rose, L. J. Chem. Soc., Chem. Commun. 1998, 33. (f) Roush, W. R.; Bennett, C. E., J. Am. Chem. Soc. 1999, 121, 3541. (g) Roush, W. R.; Gung, B. W.; Bennett, C. E. Org. Lett. 1999, 1, 891. (h) Roush, W. R.; Narayan, S.; Bennett, C. E.; Briner, K. Org. Lett. 1999, 1, 895. (i) Roush, W. R.; Narayan, S. Org. Lett. 1999, 1, 899.

19. Smith, E. M. Tetrahedron Lett. 1999, 40, 3285.

20. In analogy to tetra- $n$-butylammonium fluoride Amberlyst A-26 ( $\mathrm{F}^{-}$- form) was employed for the treatment of various silyl ethers. While both primary as well as secondary triethylsilyl groups can rapidly be removed with this polymer-bound reagent is not reliable when cleaving secondary primary TBS-ethers.

21. Nicolaou, K. C.; Winssinger, N.; Vourloumis D. Angew. Chem., Int. Ed. 2000, 39, 1.

22. Nicolaou, K. C.; Winssinger, N.; Vourloumis, D.; Ohshima, T.; Kim, S.; Pfefferkorn, J.; Xu, J.-Y.; Li, T. J. Am. Chem. Soc. 1998, 120, 10814.

23. Kubota, H.; Lim, J.; Depew, K. M.; Schreiber, S. L. Chem. Biol. 2002, 9, 265.

24. Tan, D. S.; Foley, M. A.; Stockwell, B. R.; Shair, M. D.; Schreiber, S. L. J. Am. Chem. Soc. 1999, 121, 9073. 
25. (a) Grabley, S.; Granzer, E.; Hütter, K.; Ludwig, D.; Mayer, M.; Thiericke, R.; Till, G.; Wink, J.; Phillips, S.; Zeeck, A. J. Antibiot. 1992, 45, 56. (b) Göhrt, A.; Zeeck, A.; Hütter, K.; Hirsch, R.; Kluge, H.; Thiericke, R., ibid. 1992, 45, 66.

26. Ayer, W.; Sun, M.; Browne, L. M.; Brinen, L. S.; Clardy, J. J. Nat. Prod. 1992, 55, 649.

27. (a) Dräger, G.; Kirschning, A.; Thiericke, R.; Zerlin, M., Natural Products Reports 1996, 13, 365. (b) Rousseau, G. Tetrahedron 1995, 51, 2777.

28. Kirschning, A.; Dräger, G.; Garming, A.; Thiericke, R.; Zerlin, M.; Maul, C.; Noltemeyer, M. Chem. Eur. J. 1998, 1324.

29. International Tables for Crystallography; Vol. A; (Ed.: Hahn, T.), 2nd Edn; Dordrecht, 1989.

30. Sheldrick, G. M.; SHELXS-86, Acta Crytallogr. 1990, A46, 467. 